\title{
Weak Interactions in a Background of a Uniform Magnetic Field. A Mathematical Model for the Inverse $\beta$ Decay. $I$.
}

\author{
Jean-Claude Guillot \\ Centre de Mathématiques Appliquées, École Polytechnique-C.N.R.S, Palaiseau Cedex, France \\ Email: Jean-Claude.Guillot@polytechnique.edu
}

How to cite this paper: Guillot, J.-C. (2017) Weak Interactions in a Background of a Uniform Magnetic Field. A Mathematical Model for the Inverse $\beta$ Decay. $I$. Open Access Library Journal, 4: e4142. https://doi.org/10.4236/oalib.1104142

Received: November 10, 2017

Accepted: December 16, 2017

Published: December 19, 2017

Copyright $\odot 2017$ by author and Open Access Library Inc.

This work is licensed under the Creative Commons Attribution International License (CC BY 4.0).

http://creativecommons.org/licenses/by/4.0/

\begin{abstract}
In this paper we consider a mathematical model for the inverse $\beta$ decay in a uniform magnetic field. With this model we associate a Hamiltonian with cutoffs in an appropriate Fock space. No infrared regularization is assumed. The Hamiltonian is self-adjoint and has a unique ground state. We study the essential spectrum and determine the spectrum. The coupling constant is supposed sufficiently small.
\end{abstract}

\section{Subject Areas}

Functional Analysis, Particle Physics

\section{Keywords}

Beta Decay, Uniform Magnetic Field, Weak Interactions, Spectral Theory

\section{Introduction}

A supernova is initiated by the collapse of a stellar core which leads to the formation of a protoneutron star which may be formed with strong magnetic fields typically of order $10^{16}$ Gauss. It turns out that the protoneutron star leads to the formation of a neutron star in a very short time during which almost all the gravitational binding energy of the protoneutron star is emmitted in neutrinos and antineutrinos of each type. Neutron stars have strong magnetic fields of order $10^{12}$ Gauss. Thus neutrinos interactions are of great importance because of their capacity to serve as mediators for the transport and loss of energy and the following processes, the so-called "Urca" ones or inverse $\beta$ decays in Physics, 


$$
\begin{aligned}
& v_{e}+n \rightleftharpoons e_{-}+p \\
& \overline{v_{e}}+p \rightleftharpoons e_{+}+n
\end{aligned}
$$

play an essential role in those phenomena and they are associated with the $\beta$ decay

$$
n \rightarrow p+e_{+}+\overline{v_{e}}
$$

Here $e_{-}$(resp. $e_{+}$) is an electron (resp. a positron). $p$ is a proton and $n$ a neutron. $v_{e}$ and $\overline{v_{e}}$ are the neutrino and the antineutrino associated with the electron.

See [1] [2] [3] [4] and references therein.

We only consider here high-energy neutrinos and antineutrinos which are indeed relativistic particles whose mass is zero or in anyway negligible.

Due to the large magnetic field strengths involved, it is quite fundamental to study the processes (1.1) and (1.2) in the presence of magnetic fields.

These realistic fields may be very complicated in their structure but we assume these fields to be locally uniform which is a very good hypothesis because the range of the weak interactions is very short. Our aim is to study the processes (1.1) and (1.2) in a background of a uniform magnetic field.

Throughout this work we restrict ourselves to the study of processes (1.1), the study of processes (1.2) and (1.3) would be quite similar. We choose the units such that $c=\hbar=1$.

The advantage of a uniform magnetic field is that, in presence of this field, Dirac equation can be exactly solved. Using the generalized eigenfunctions of the Dirac equation and the canonical quantization we carefully define the quantized fields associated with the electrons, the positrons, the protons and the antiprotons in a uniform magnetic field.

For the neutrons and the neutrinos we define the corresponding quantized fields by using the helicity formalism for the free Dirac equation.

We then consider the Fock space for the electrons, the positrons, the protons, the antiprotons, the neutrons and the neutrinos.

In this paper we consider a mathematical model for the process (1.1) in a uniform magnetic field based on the Fermi's Hamiltonian for the $\beta$ decay. The physical interaction is a highly singular operator due to delta-distributions associated with the conservation of momenta and because of the ultraviolet divergences. In order to get a well defined Hamiltonian in the Fock space we have to substitute smoother kernels both for the delta-distributions and for dealing with the ultraviolet divergences. We then get a self-adjoint Hamiltonian with cutoffs in the Fock space when the kernels are square integrable.

We then study the essential spectrum of the Hamiltonian and prove the existence of a unique ground state with appropriate hypothesis on the kernels. The proof of the uniqueness of the ground state is a direct consequence of the proof of the existence of a ground state. The spectrum of the Hamiltonian is identical to its essential spectrum. Every result is obtained for a sufficiently small 
coupling constant. No infrared regularization is assumed. We adapt to our case the proofs given in [5] and [6].

These results are new for the mathematical models in Quantum Field Theory with a uniform magnetic field.

The paper is organized as follows. In the next two sections, we quantize the Dirac fields for electrons, protons and their antiparticles in a uniform magnetic field. In the third section, we quantize the Dirac fields for free neutrons, neutrinos and their antiparticles in helicity formalism. The self-adjoint Hamiltonian of the model is defined in the fourth section. We then study the essential spectrum and prove the existence of a unique ground state.

\section{The Quantization of the Dirac Fields for the Electrons and the Protons in a Uniform Magnetic Field}

In this paper we assume that the uniform classical background magnetic field in $\mathbb{R}^{3}$ is along the $x^{3}$-direction of the coordinate axis. There are several choices of gauge vector potential giving rise to a magnetic field of magnitude $B>0$ along the $x^{3}$-direction. In this paper we choose the following vector potential

$$
\begin{aligned}
& \mathbf{A}(\mathbf{x})=\left(A^{\mu}(\mathbf{x})\right), \mu=0,1,2,3, \text { where } \\
& A^{0}(\mathbf{x})=A^{2}(\mathbf{x})=A^{3}(\mathbf{x})=0, A^{1}(\mathbf{x})=-x^{2} B
\end{aligned}
$$

Here $\boldsymbol{x}=\left(x^{1}, x^{2}, x^{3}\right)$ in $\mathbb{R}^{3}$.

We recall that we neglect the anomalous magnetic moments of the particles of $\operatorname{spin} \frac{1}{2}$.

The Dirac equation for a particle of $\operatorname{spin} \frac{1}{2}$ with mass $m>0$ and charge $e$ in a uniform magnetic field of magnitude $B>0$ along the $x^{3}$-direction with the choice of the gauge (2.1) and by neglecting its anomalous magnetic moment is given by

$$
H_{D}(e)=\alpha \cdot\left(\frac{1}{i} \nabla-e \mathbf{A}\right)+\beta m
$$

acting in the Hilbert space $L^{2}\left(\mathbb{R}^{3}, \mathbb{C}^{4}\right)$.

The scalar product in $L^{2}\left(\mathbb{R}^{3}, \mathbb{C}^{4}\right)$ is given by

$$
(f, g)=\sum_{j=1}^{4} \int_{\mathbb{R}^{3}} \overline{f(\mathbf{x})_{j}} g(\mathbf{x})_{j} \mathrm{~d}^{3} \mathbf{x}
$$

We refer to [7] for a discussion of the Dirac operator.

Here $\boldsymbol{\alpha}=\left(\alpha_{1}, \alpha_{2}, \alpha_{3}\right), \quad \beta$ are the Dirac matrices in the standard form:

$$
\beta=\left(\begin{array}{cc}
I & 0 \\
0 & -I
\end{array}\right), \quad \alpha_{i}=\left(\begin{array}{cc}
0 & \sigma_{i} \\
\sigma_{i} & 0
\end{array}\right), \quad i=1,2,3
$$

where $\sigma_{i}$ are the usual Pauli matrices.

By ([7], thm 4.3) $H_{D}(e)$ is essentially self-adjoint on $C_{0}^{\infty}\left(\mathbb{R}^{3}, \mathbb{C}^{4}\right)$. The spectrum of $H_{D}(e)$ is equal to 


$$
\operatorname{spec}\left(H_{D}(e)\right)=(-\infty,-m] \cup[m, \infty]
$$

The spectrum of $H_{D}(e)$ is absolutely continuous and its multiplicity is not uniform. There is a countable set of thresholds, denoted by $S$, where

$$
S=\left(-S_{n}, s_{n} ; n \in \mathbb{N}\right)
$$

with $s_{n}=\sqrt{m^{2}+2 n|e| B}$. See [8].

We consider a spectral representation of $H_{D}(e)$ based on a complete set of generalized eigenfunctions of the continuous spectrum of $H_{D}(e)$. Those generalized eigenfunctions are well known. See [9]. In view of (2.1) we use the computation of the generalized eigenfunctions given by [10] and [11]. See also [4] and references therein.

Let $\left(p^{1}, p^{3}\right)$ be the conjugate variables of $\left(x^{1}, x^{3}\right)$. By the Fourier transform in $\mathbb{R}^{2}$ we easily get

$$
L^{2}\left(\mathbb{R}^{3}, \mathbb{C}^{4}\right) \simeq \int_{\mathbb{R}^{2}}^{\oplus} L^{2}\left(\mathbb{R}, \mathbb{C}^{4}\right) \mathrm{d} p^{1} \mathrm{~d} p^{3}
$$

and

$$
H_{D}(e) \simeq \int_{\mathbb{R}^{2}}^{\oplus} H_{D}\left(e ; p^{1}, p^{3}\right) \mathrm{d} p^{1} \mathrm{~d} p^{3}
$$

where

$$
\begin{aligned}
& H_{D}\left(e ; p^{1}, p^{3}\right) \\
& =\left(\begin{array}{cc}
m \sigma_{0} & \sigma_{1}\left(p^{1}-e x^{2} B\right)-i \sigma_{2} \frac{\mathrm{d}}{\mathrm{d} x^{2}}+p^{3} \sigma_{3} \\
\sigma_{1}\left(p^{1}-e x^{2} B\right)-i \sigma_{2} \frac{\mathrm{d}}{\mathrm{d} x^{2}}+p^{3} \sigma_{3} & -m \sigma_{0}
\end{array}\right)
\end{aligned}
$$

Here $\sigma_{0}$ is the $2 \times 2$ unit matrix.

$H_{D}\left(e ; p^{1}, p^{3}\right)$ is the reduced Dirac operator associated to $\left(e ; p^{1}, p^{3}\right)$.

$H_{D}\left(e ; p^{1}, p^{3}\right)$ is essentially self-adjoint on $C_{0}^{\infty}\left(\mathbb{R}, \mathbb{C}^{4}\right)$ and has a pure point spectrum which is symmetrical with respect to the origin.

Set

$$
E_{n}\left(p^{3}\right)^{2}=m^{2}+\left(p^{3}\right)^{2}+2 n|e| B, n \geq 0
$$

The positive spectrum of $H_{D}\left(e ; p^{1}, p^{3}\right)$ is the set of eigenvalues $\left(E_{n}\left(p^{3}\right)\right)_{n \geq 0}$ and the negative spectrum is the set of eigenvalues $\left(-E_{n}\left(p^{3}\right)\right)_{n \geq 0} \cdot E_{0}\left(p^{3}\right)$ and $-E_{0}\left(p^{3}\right)$ are simple eigenvalues and the multiplicity of $E_{n}\left(p^{3}\right)^{0}$ and $-E_{n}\left(p^{3}\right)$ is equal to 2 for $n \geq 1$.

Through out this work $e$ will be the positive unit of charge taken to be equal to the proton charge.

We now give the eigenfunctions of $H_{D}\left(e ; p^{1}, p^{3}\right)$ both for the electrons and for the protons. The eigenfunctions are labelled by $n \in \mathbb{N},\left(p_{1}, p_{2}\right) \in \mathbb{R}^{2}$ and $s= \pm 1 . n \in \mathbb{N}$ labels the nth Landau level. $s= \pm 1$ are the eigenvalues of $\sigma_{3}$. The electrons and the protons in all Landau levels with $n \geq 1$ can have different spin polarizations $s= \pm 1$. However in the lowest Landau state $n=0$ the electrons can only have the spin orientation given by $s=-1$ and the protons 
can only have the spin orientation given by $s=1$.

\subsection{Eigenfunctions of the Reduced Dirac Operator for the Electrons}

We now compute the eigenfunctions of $H_{D}\left(-e ; p^{1}, p^{3}\right)$ with $m=m_{e}$ where $m_{e}$ is the mass of the electron.

$E_{n}^{(e)}\left(p^{3}\right)$ and $-E_{n}^{(e)}\left(p^{3}\right)$ will denote the eigenvalues of $H_{D}\left(-e ; p^{1}, p^{3}\right)$ for the electrons. We have $E_{n}^{(e)}\left(p^{3}\right)^{2}=m_{e}^{2}+\left(p^{3}\right)^{2}+2 n e B, n \geq 0$.

\subsubsection{Eigenfunctions of the Electrons for Positive Eigenvalues}

For $n \geq 1 E_{n}^{(e)}\left(p^{3}\right)$ is of multiplicity two corresponding to $s= \pm 1$ and $E_{0}^{(e)}\left(p^{3}\right)$ is multiplicity one corresponding to $s=-1$.

Let $U_{ \pm 1}^{(e)}\left(x^{2}, n, p^{1}, p^{3}\right)$ denote the eigenfunctions associated to $s= \pm 1$.

For $s=1$ and $n \geq 1$ we have

$$
U_{+1}^{(e)}\left(x^{2}, n, p^{1}, p^{3}\right)=\left(\frac{E_{n}^{(e)}\left(p^{3}\right)+m_{e}}{2 E_{n}^{(e)}\left(p^{3}\right)}\right)^{\frac{1}{2}}\left(\begin{array}{c}
I_{n-1}(\xi) \\
0 \\
\frac{p^{3}}{E_{n}^{(e)}\left(p^{3}\right)+m_{e}} I_{n-1}(\xi) \\
-\frac{\sqrt{2 n e B}}{E_{n}^{(e)}\left(p^{3}\right)+m_{e}} I_{n}(\xi)
\end{array}\right)
$$

where

$$
\begin{aligned}
& \xi=\sqrt{e B}\left(x^{2}-\frac{p^{1}}{e B}\right) \\
& I_{n}(\xi)=\left(\frac{\sqrt{e B}}{n ! 2^{n} \sqrt{\pi}}\right)^{\frac{1}{2}} \exp \left(-\xi^{2} / 2\right) H_{n}(\xi)
\end{aligned}
$$

Here $H_{n}(\xi)$ is the Hermite polynomial of order $\mathrm{n}$ and we define

$$
I_{-1}(\xi)=0
$$

For $n=0$ and $s=1$ we set

$$
U_{+1}^{(e)}\left(x^{2}, 0, p^{1}, p^{3}\right)=0
$$

For $s=-1$ and $n \geq 0$ we have

$$
U_{-1}^{(e)}\left(x^{2}, n, p^{1}, p^{3}\right)=\left(\frac{E_{n}^{(e)}\left(p^{3}\right)+m_{e}}{2 E_{n}^{(e)}\left(p^{3}\right)}\right)^{\frac{1}{2}}\left(\begin{array}{c}
0 \\
I_{n}(\xi) \\
-\frac{\sqrt{2 n e B}}{E_{n}^{(e)}\left(p^{3}\right)+m_{e}} I_{n-1}(\xi) \\
-\frac{p^{3}}{E_{n}^{(e)}\left(p^{3}\right)+m_{e}} I_{n}(\xi)
\end{array}\right)
$$

Note that

$$
\int \mathrm{d} x^{2} U_{s}^{(e)}\left(x^{2}, n, p^{1}, p^{3}\right)^{\dagger} U_{s^{\prime}}^{(e)}\left(x^{2}, n, p^{1}, p^{3}\right)=\delta_{s s^{\prime}}
$$


where $\dagger$ is the adjoint in $\mathbb{C}^{4}$.

\subsubsection{Eigenfunctions of the Electrons for Negative Eigenvalues}

For $n \geq 1-E_{n}^{(e)}\left(p^{3}\right)$ is of multiplicity two corresponding to $s= \pm 1$ and $-E_{0}^{(e)}\left(p^{3}\right)$ is multiplicity one corresponding to $s=-1$.

Let $V_{ \pm 1}^{(e)}\left(x^{2}, n, p^{1}, p^{3}\right)$ denote the eigenfunctions associated with the eigenvalue $-E_{n}^{(a p)}\left(p^{3}\right)$ and with $s= \pm 1$.

For $s=1$ and $n \geq 1$ we have

$$
V_{+1}^{(e)}\left(x^{2}, n, p^{1}, p^{3}\right)=\left(\frac{E_{n}^{(e)}\left(p^{3}\right)+m_{e}}{2 E_{n}^{(e)}\left(p^{3}\right)}\right)^{\frac{1}{2}}\left(\begin{array}{c}
-\frac{p^{3}}{E_{n}^{(e)}\left(p^{3}\right)+m_{e}} I_{n-1}(\xi) \\
\frac{\sqrt{2 n e B}}{E_{n}^{(e)}\left(p^{3}\right)+m_{e}} I_{n}(\xi) \\
I_{n-1}(\xi) \\
0
\end{array}\right)
$$

and for $n=0$ we set

$$
V_{+1}^{(e)}\left(x^{2}, 0, p^{1}, p^{3}\right)=0
$$

For $s=-1$ and $n \geq 0$ we have

$$
V_{-1}^{(e)}\left(x^{2}, n, p^{1}, p^{3}\right)=\left(\frac{E_{n}^{(e)}\left(p^{3}\right)+m_{e}}{2 E_{n}^{(e)}\left(p^{3}\right)}\right)^{\frac{1}{2}}\left(\begin{array}{c}
\frac{\sqrt{2 n e B}}{E_{n}^{(e)}\left(p^{3}\right)+m_{e}} I_{n-1}(\xi) \\
\frac{p^{3}}{E_{n}^{(e)}\left(p^{3}\right)+m_{e}} I_{n}(\xi) \\
0 \\
I_{n}(\xi)
\end{array}\right)
$$

Note that

$$
\int \mathrm{d} x^{2} V_{s}^{(e)}\left(x^{2}, n, p^{1}, p^{3}\right)^{\dagger} V_{s^{\prime}}^{(e)}\left(x^{2}, n, p^{1}, p^{3}\right)=\delta_{s s^{\prime}}
$$

where $\dagger$ is the adjoint in $\mathbb{C}^{4}$.

The sets $\left(U_{ \pm 1}^{(e)}\left(., n, p^{1}, p^{3}\right)\right)_{\left(n, p^{1}, p^{3}\right)}$ and $\left(V_{ \pm 1}^{(e)}\left(., n, p^{1}, p^{3}\right)\right)_{\left(n, p^{1}, p^{3}\right)}$ of vectors in $L^{2}\left(\mathbb{R}, \mathbb{C}^{4}\right)$ form a orthonormal basis of $L^{2}\left(\mathbb{R}, \mathbb{C}^{4}\right)$.

This yields for $\Psi(\mathbf{x})$ in $L^{2}\left(\mathbb{R}^{3}, \mathbb{C}^{4}\right)$

$$
\begin{aligned}
\Psi(\mathbf{x})= & \frac{1}{2 \pi} \sum_{s= \pm 1} \operatorname{Li} . \mathrm{m}\left(\sum _ { n \geq 0 } \int _ { \mathbb { R } ^ { 2 } } \mathrm { d } p ^ { 1 } \mathrm { d } p ^ { 3 } \mathrm { e } ^ { i ( p ^ { 1 } x ^ { 1 } + p ^ { 3 } x ^ { 3 } ) } \left(c_{s}^{(e)}\left(n, p^{1}, p^{3}\right) U_{s}^{(e)}\left(x^{2}, n, p^{1}, p^{3}\right)\right.\right. \\
& \left.\left.+d_{s}^{(e)}\left(n, p^{1}, p^{3}\right) V_{s}^{(e)}\left(x^{2}, n, p^{1}, p^{3}\right)\right)\right)
\end{aligned}
$$

where $c_{+1}^{(e)}\left(0, p^{1}, p^{3}\right)=d_{+1}^{(e)}\left(0, p^{1}, p^{3}\right)=0$.

Let $\hat{\Psi}\left(x^{2} ; p^{1}, p^{3}\right)$ be the Fourier transform of $\Psi($.$) with respect to x^{1}$ and $x^{3}$ :

$$
\hat{\Psi}\left(x^{2} ; p^{1}, p^{3}\right)=\text { L.i.m } \frac{1}{2 \pi} \int_{\mathbb{R}^{2}} e^{-i\left(p^{1} x^{1}+p^{3} x^{3}\right)} \Psi\left(x^{1}, x^{2}, x^{3}\right) \mathrm{d} x^{1} \mathrm{~d} x^{3}
$$


We have

$$
\begin{aligned}
& C_{s}^{(e)}\left(n, p^{1}, p^{3}\right)=\int_{\mathbb{R}} U_{s}^{(e)}\left(x^{2}, n, p^{1}, p^{3}\right)^{\dagger} \hat{\Psi}\left(x^{2} ; p^{1}, p^{3}\right) \mathrm{d} x^{2} \\
& d_{s}^{(e)}\left(n, p^{1}, p^{3}\right)=\int_{\mathbb{R}} V_{s}^{(e)}\left(x^{2}, n, p^{1}, p^{3}\right)^{\dagger} \hat{\Psi}\left(x^{2} ; p^{1}, p^{3}\right) \mathrm{d} x^{2}
\end{aligned}
$$

The complex coefficients $c_{s}^{(e)}\left(n, p^{1}, p^{3}\right)$ and $d_{s}^{(e)}\left(n, p^{1}, p^{3}\right)$ satisfy

$$
\|\Psi(.)\|_{L^{2}\left(\mathbb{R}^{3}, \mathbb{C}^{4}\right)}^{2}=\sum_{s= \pm 1} \sum_{n \geq 0} \int\left(\left|c_{s}^{(e)}\left(n, p^{1}, p^{3}\right)\right|^{2}+\left|d_{s}^{(e)}\left(n, p^{1}, p^{3}\right)\right|^{2}\right) \mathrm{d} p^{1} \mathrm{~d} p^{3}<\infty
$$

\subsection{Eigenfunctions of the Reduced Dirac Operator for the Protons}

We now compute the eigenfunctions of $H_{D}\left(e ; p^{1}, p^{3}\right)$ with $m=m_{p}$.

$E_{n}^{(p)}\left(p^{3}\right)$ and $-E_{n}^{(p)}\left(p^{3}\right)$ denote the eigenvalues of $H_{D}\left(e ; p^{1}, p^{3}\right)$ for the proton. We have $E_{n}^{(p)}\left(p^{3}\right)^{2}=m_{p}^{2}+\left(p^{3}\right)^{2}+2 n e B, n \geq 0$.

\subsubsection{Eigenfunctions of the Proton for Positive Eigenvalues}

For $n \geq 1 E_{n}^{(p)}\left(p^{3}\right)$ is of multiplicity two corresponding to $s= \pm 1$ and $E_{0}^{(p)}\left(p^{3}\right)$ is of multiplicity one corresponding to $s=1$.

Let $U_{ \pm 1}^{(p)}\left(x^{2}, n, p^{1}, p^{3}\right)$ denote the eigenfunctions associated with the eigenvalue $E_{n}^{(p)}\left(p^{3}\right)$ and with $s= \pm 1$.

For $s=1$ and $n \geq 0$ we have

$$
U_{+1}^{(p)}\left(x^{2}, n, p^{1}, p^{3}\right)=\left(\frac{E_{n}^{(p)}\left(p^{3}\right)+m_{p}}{2 E_{n}^{(p)}\left(p^{3}\right)}\right)^{\frac{1}{2}}\left(\begin{array}{c}
I_{n}(\tilde{\xi}) \\
0 \\
\frac{p^{3}}{E_{n}^{(p)}\left(p^{3}\right)+m_{p}} I_{n}(\tilde{\xi}) \\
\frac{\sqrt{2 n e B}}{E_{n}^{(p)}\left(p^{3}\right)+m_{p}} I_{n-1}(\tilde{\xi})
\end{array}\right)
$$

where

$$
\begin{aligned}
& \tilde{\xi}=\sqrt{e B}\left(x^{2}+\frac{p^{1}}{e B}\right) \\
& I_{-1}(\tilde{\xi})=0
\end{aligned}
$$

For $s=-1$ and $n \geq 1$ we have

$$
U_{-1}^{(p)}\left(x^{2}, n, p^{1}, p^{3}\right)=\left(\frac{E_{n}^{(p)}\left(p^{3}\right)+m_{p}}{2 E_{n}^{(p)}\left(p^{3}\right)}\right)^{\frac{1}{2}}\left(\begin{array}{c}
0 \\
I_{n-1}(\tilde{\xi}) \\
\frac{\sqrt{2 n e B}}{E_{n}^{(p)}\left(p^{3}\right)+m_{p}} I_{n}(\tilde{\xi}) \\
-\frac{p^{3}}{E_{n}^{(p)}\left(p^{3}\right)+m_{p}} I_{n-1}(\tilde{\xi})
\end{array}\right)
$$

For $n=0$ and $s=-1$ we set

$$
U_{-1}^{(p)}\left(x^{2}, 0, p^{1}, p^{3}\right)=0
$$


Note that

$$
\int \mathrm{d} x^{2} U_{s}^{(p)}\left(x^{2}, n, p^{1}, p^{3}\right)^{\dagger} U_{s^{\prime}}^{(p)}\left(x^{2}, n, p^{1}, p^{3}\right)=\delta_{s s^{\prime}}
$$

where $\dagger$ is the adjoint in $\mathbb{C}^{4}$.

\subsubsection{Eigenfunctions of the Proton for Negative Eigenvalues}

For $n \geq 1-E_{n}^{(p)}\left(p^{3}\right)$ is of multiplicity two corresponding to $s= \pm 1$ and $-E_{0}^{(p)}\left(p^{3}\right)$ is of multiplicity one corresponding to $s=1$.

Let $V_{ \pm 1}^{(p)}\left(x^{2}, n, p^{1}, p^{3}\right)$ denote the eigenfunctions associated with the eigenvalue $-E_{n}^{(p)}\left(p^{3}\right)$ and with $s= \pm 1$.

For $s=1$ and $n \geq 0$ we have

$$
V_{+1}^{(p)}\left(x^{2}, n, p^{1}, p^{3}\right)=\left(\frac{E_{n}^{(p)}\left(p^{3}\right)+m_{p}}{2 E_{n}^{(p)}\left(p^{3}\right)}\right)^{\frac{1}{2}}\left(\begin{array}{c}
-\frac{p^{3}}{E_{n}^{(p)}\left(p^{3}\right)+m_{p}} I_{n-1}(\tilde{\xi}) \\
-\frac{\sqrt{2 n e B}}{E_{n}^{(p)}\left(p^{3}\right)+m_{p}} I_{n-1}(\tilde{\xi}) \\
I_{n}(\tilde{\xi}) \\
0
\end{array}\right)
$$

For $s=-1$ and $n \geq 1$ we have

$$
V_{-1}^{(p)}\left(x^{2}, n, p^{1}, p^{3}\right)=\left(\frac{E_{n}^{(p)}\left(p^{3}\right)+m_{p}}{2 E_{n}^{(p)}\left(p^{3}\right)}\right)^{\frac{1}{2}}\left(\begin{array}{c}
-\frac{\sqrt{2 n e B}}{E_{n}^{(p)}\left(p^{3}\right)+m_{p}} I_{n}(\tilde{\xi}) \\
\frac{p^{3}}{E_{n}^{(p)}\left(p^{3}\right)+m_{p}} I_{n-1}(\tilde{\xi}) \\
0 \\
I_{n-1}(\tilde{\xi})
\end{array}\right)
$$

and for $n=0$ and $s=-1$ we set

$$
V_{-1}^{(p)}\left(x^{2}, 0, p^{1}, p^{3}\right)=0
$$

Note that

$$
\int \mathrm{d} x^{2} V_{s}^{(p)}\left(x^{2}, n, p^{1}, p^{3}\right)^{\dagger} V_{s^{\prime}}^{(p)}\left(x^{2}, n, p^{1}, p^{3}\right)=\delta_{s s^{\prime}}
$$

where $\dagger$ is the adjoint in $\mathbb{C}^{4}$.

The sets $\left(U_{ \pm 1}^{(p)}\left(., n, p^{1}, p^{3}\right)\right)_{\left(n, p^{1}, p^{3}\right)}$ and $\left(V_{ \pm 1}^{(p)}\left(., n, p^{1}, p^{3}\right)\right)_{\left(n, p^{1}, p^{3}\right)}$ of vectors in $L^{2}\left(\mathbb{R}, \mathbb{C}^{4}\right)$ form an orthonormal basis of $L^{2}\left(\mathbb{R}, \mathbb{C}^{4}\right)$.

This yields for $\Psi(\mathbf{x})$ in $L^{2}\left(\mathbb{R}^{3}, \mathbb{C}^{4}\right)$

$$
\begin{aligned}
\Psi(\mathbf{x})= & \frac{1}{2 \pi} \sum_{s= \pm 1} \text { L.i.m }\left(\sum _ { n \geq 0 } \int _ { \mathbb { R } ^ { 2 } } \mathrm { d } p ^ { 1 } \mathrm { d } p ^ { 3 } \mathrm { e } ^ { i ( p ^ { 1 } x ^ { 1 } + p ^ { 3 } x ^ { 3 } ) } \left(c_{s}^{(p)}\left(n, p^{1}, p^{3}\right) U_{s}^{(p)}\left(x^{2}, n, p^{1}, p^{3}\right)\right.\right. \\
& \left.\left.+d_{s}^{(p)}\left(n, p^{1}, p^{3}\right) V_{s}^{(p)}\left(x^{2}, n, p^{1}, p^{3}\right)\right)\right)
\end{aligned}
$$

where $c_{-1}^{(p)}\left(0, p^{1}, p^{3}\right)=d_{-1}^{(p)}\left(0, p^{1}, p^{3}\right)=0$ 
The complex coefficients $c_{s}^{(p)}\left(n, p^{1}, p^{3}\right)$ and $d_{s}^{(p)}\left(n, p^{1}, p^{3}\right)$ satisfy

$\|\Psi(.)\|_{L^{2}\left(\mathbb{R}^{3}, \mathbb{C}^{4}\right)}^{2}=\sum_{s= \pm 1 n \geq 0} \int\left(\left(\left|c_{s}^{(p)}\left(n, p^{1}, p^{3}\right)\right|^{2}+\left|d_{s}^{(p)}\left(n, p^{1}, p^{3}\right)\right|^{2}\right) \mathrm{d} p^{1} \mathrm{~d} p^{3}\right)<\infty$

We have

$$
\begin{aligned}
& c_{s}^{(p)}\left(n, p^{1}, p^{3}\right)=\int_{\mathbb{R}} U_{s}^{(p)}\left(x^{2}, n, p^{1}, p^{3}\right)^{\dagger} \hat{\Psi}\left(x^{2} ; p^{1}, p^{3}\right) \mathrm{d} x^{2} \\
& d_{s}^{(p)}\left(n, p^{1}, p^{3}\right)=\int_{\mathbb{R}} V_{s}^{(p)}\left(x^{2}, n, p^{1}, p^{3}\right)^{\dagger} \hat{\Psi}\left(x^{2} ; p^{1}, p^{3}\right) \mathrm{d} x^{2}
\end{aligned}
$$

\subsubsection{Eigenfunctions of the Positron for Positive Eigenvalues}

The generalized eigenfunctions for the positron, denoted by $U_{ \pm 1}^{(-e)}\left(x^{2}, n, p^{1}, p^{3}\right)$, are obtained from $U_{ \pm 1}^{(p)}\left(x^{2}, n, p^{1}, p^{3}\right)$ by substituting the mass of the electron $m_{e}$ for $m_{p}$. The associated eigenvalues are denoted by $E_{n}^{(-e)}\left(p^{3}\right)$ with $E_{n}^{(-e)}\left(p^{3}\right)^{2}=m_{e}^{2}+\left(p^{3}\right)^{2}+2 n e B, n \geq 0$.

\subsubsection{Eigenfunctions of the Positron for Negative Eigenvalues}

The generalized eigenfunctions for the positron, associated with the eigenvalues $-E_{n}^{(-e)}\left(p^{3}\right)$ and denoted by $V_{ \pm 1}^{(-e)}\left(x^{2}, n, p^{1}, p^{3}\right)$, are obtained from $V_{ \pm 1}^{(p)}\left(x^{2}, n, p^{1}, p^{3}\right)$ by substituting the mass of the electron $m_{e}$ for $m_{p}$.

\subsubsection{Eigenfunctions of the Antiproton for Positive Eigenvalues}

The generalized eigenfunctions for the antiproton, denoted by $U_{ \pm 1}^{(-p)}\left(x^{2}, n, p^{1}, p^{3}\right)$, are obtained from $U_{ \pm 1}^{(e)}\left(x^{2}, n, p^{1}, p^{3}\right)$ by substituting the mass of the proton $m_{p}$ for $m_{e}$. The associated eigenvalues are denoted by $E_{n}^{(-p)}\left(p^{3}\right)$ with $E_{n}^{(-p)}\left(p^{3}\right)^{2}=m_{p}^{2}+\left(p^{3}\right)^{2}+2 n e B, n \geq 0$.

\subsubsection{Eigenfunctions of the Antiproton for Negative Eigenvalues}

The generalized eigenfunctions for the antiproton, associated with the eigenvalues $-E_{n}^{(-p)}\left(p^{3}\right)$ and denoted by $V_{ \pm 1}^{(-p)}\left(x^{2}, n, p^{1}, p^{3}\right)$, are obtained from $V_{ \pm 1}^{(e)}\left(x^{2}, n, p^{1}, p^{3}\right)$ by substituting the mass of the proton $m_{p}$ for $m_{e}$.

\subsection{Fock Spaces for Electrons, Positrons, Protons and Antiprotons in a Uniform Magnetic Field}

It follows from Sections 2.1 and 2.2 that $\left(s, n, p^{1}, p^{3}\right)$ are quantum variables for the electrons, the positrons, the protons and the antiprotons in a uniform magnetic field.

Let $\xi_{1}=\left(s, n, p_{e}^{1}, p_{e}^{3}\right)$ be the quantum variables of a electron and of a positron and let $\xi_{2}=\left(s, n, p_{p}^{1}, p_{p}^{3}\right)$ be the quantum variables of a proton and of an antiproton.

We set $\Gamma_{1}=\{-1,1\} \times \mathbb{N} \times \mathbb{R}^{2}$ for the configuration space for both the electrons, the positrons, the protons and the antiprotons. $L^{2}\left(\Gamma_{1}\right)$ is the Hilbert space associated to each species of fermions.

We have, by (2.17), (2.18), (2.19), (2.26), (2.27) and (2.28),

$$
L^{2}\left(\Gamma_{1}\right)=l^{2}\left(L^{2}\left(\mathbb{R}^{2}\right)\right) \oplus I^{2}\left(L^{2}\left(\mathbb{R}^{2}\right)\right)
$$

Let $\mathfrak{F}_{(e)}$ and $\mathfrak{F}_{(-e)}$ denote the Fock spaces for the electrons and the posi- 
trons respectively and let $\mathfrak{F}_{(p)}$ and $\mathfrak{F}_{(-p)}$ denote the Fock spaces for the protons and the antiprotons respectively.

We have

$$
\mathfrak{F}_{(e)}=\mathfrak{F}_{(-e)}=\mathfrak{F}_{(p)}=\mathfrak{F}_{(-p)}=\bigoplus_{n=0}^{\infty} \underset{a}{\otimes} L^{2}\left(\Gamma_{1}\right)
$$

$\bigotimes_{a}^{n} L^{2}\left(\Gamma_{1}\right)$ is the antisymmetric $n$-th tensor power of $L^{2}\left(\Gamma_{1}\right)$.

$\Omega_{(\alpha)}=(1,0,0,0, \cdots)$ is the vacuum state in $\mathfrak{F}_{(\alpha)}$ for $\alpha=e,-e, p,-p$.

We shall use the notations

$$
\begin{aligned}
& \int_{\Gamma_{1}} \mathrm{~d} \xi_{1}=\sum_{s= \pm 1 n \geq 0} \int_{\mathbb{R}^{2}} \mathrm{~d} p_{e}^{1} \mathrm{~d} p_{e}^{3} \\
& \int_{\Gamma_{1}} \mathrm{~d} \xi_{2}=\sum_{s= \pm 1 n \geq 0} \int_{\mathbb{R}^{2}} \mathrm{~d} p_{p}^{1} \mathrm{~d} p_{p}^{3}
\end{aligned}
$$

Set $\epsilon= \pm$.

$b_{\epsilon}\left(\xi_{j}\right)$ (resp. $b_{\epsilon}^{*}\left(\xi_{j}\right)$ ) are the annihilation (resp.creation) operators for the electron when $j=1$ and for the proton when $j=2$ if $\epsilon=+$.

$b_{\epsilon}\left(\xi_{j}\right)$ (resp. $b_{\epsilon}^{*}\left(\xi_{j}\right)$ ) are the annihilation (resp.creation) operators for the positron when $j=1$ and for the antiproton when $j=2$ if $\epsilon=-$.

The operators $b_{\epsilon}\left(\xi_{j}\right)$ and $b_{\epsilon}^{*}\left(\xi_{j}\right)$ fulfil the usual anticommutation relations (CAR)(see [12]).

In addition, following the convention described in ([12], Section 4.1) and ([12], Section 4.2), we assume that the fermionic creation and annihilation operators of different species of particles anticommute (see [13] arXiv for explicit definitions). In our case this property will be verified by the creation and annihilation operators for the electrons, the protons, the neutrons, the neutrinos and their respective antiparticles.

Therefore the following anticommutation relations hold for $j=1,2$

$$
\begin{aligned}
& \left\{b_{\epsilon}\left(\xi_{j}\right), b_{\epsilon^{\prime}}^{*}\left(\xi_{j}^{\prime}\right)\right\}=\delta_{\epsilon \epsilon^{\prime}} \delta\left(\xi_{j}-\xi_{j}^{\prime}\right), \\
& \left\{b_{\epsilon}^{\#}\left(\xi_{1}\right), b_{\epsilon^{\prime}}^{\#}\left(\xi_{2}\right)\right\}=0
\end{aligned}
$$

where $\left\{b, b^{\prime}\right\}=b b^{\prime}+b^{\prime} b$ and $b^{\#}=b$ or $b^{*}$.

Recall that for $\varphi \in L^{2}\left(\Gamma_{1}\right)$, the operators

$$
\begin{aligned}
b_{j, \epsilon}(\varphi) & =\int_{\Gamma_{1}} b_{\epsilon}\left(\xi_{j}\right) \overline{\varphi\left(\xi_{j}\right)} \mathrm{d} \xi_{j} . \\
b_{j, \epsilon}^{*}(\varphi) & =\int_{\Gamma_{1}} b_{\epsilon}^{*}\left(\xi_{j}\right) \varphi\left(\xi_{j}\right) \mathrm{d} \xi_{j}
\end{aligned}
$$

are bounded operators on $\mathfrak{F}_{(e)}$ and $\mathfrak{F}_{(-e)}$ for $j=1$ and on $\mathfrak{F}_{(p)}$ and $\mathfrak{F}_{(-p)}$ for $j=2$ respectively satisfying

$$
\left\|b_{j, \epsilon}^{\#}(\varphi)\right\|=\|\varphi\|_{L^{2}}
$$

\subsection{Quantized Dirac Fields for the Electrons and the Protons in a Uniform Magnetic Field}

We now consider the canonical quantization of the two classical fields (2.17) and (2.26). 
Recall that the charge conjugation operator $C$ is given, for every $\Psi(\mathbf{x})$, by

$$
C\left(\begin{array}{c}
\Psi_{1}(\mathbf{x}) \\
\Psi_{2}(\mathbf{x}) \\
\Psi_{3}(\mathbf{x}) \\
\Psi_{4}(\mathbf{x})
\end{array}\right)=\left(\begin{array}{c}
-\Psi_{4}^{*}(\mathbf{x}) \\
\Psi_{3}^{*}(\mathbf{x}) \\
\Psi_{2}^{*}(\mathbf{x}) \\
-\Psi_{1}^{*}(\mathbf{x})
\end{array}\right)
$$

Here $*$ is the complex conjugation.

Let $\Psi($.$) be locally in the domain of H_{D}(e)$. We have

$$
H_{D}(-e) C \Psi=E C \Psi \text { if } H_{D}(e) \Psi=-E \Psi
$$

(2.36) shows that, by applying the charge conjugation (2.35) to a solution of the Dirac equation with a negative energy for some particle, we get a solution of the Dirac equation for the antiparticle with a positive energy.

Thus, by applying the charge conjugation (2.35) to (2.14), (2.15), (2.23) and (2.24) which are solutions of the Dirac equation for the electrons and protons with a negative energy, we obtain

$$
\begin{aligned}
& \left(C V_{+1}^{(e)}\right)\left(x^{2}, n, p^{1}, p^{3}\right)=U_{-1}^{(-e)}\left(x^{2}, n,-p^{1},-p^{3}\right) \quad \text { for } n \geq 1 \\
& \left(C V_{-1}^{(e)}\right)\left(x^{2}, n, p^{1}, p^{3}\right)=-U_{+1}^{(-e)}\left(x^{2}, n,-p^{1},-p^{3}\right) \text { for } n \geq 0 \\
& \left(C V_{+1}^{(p)}\right)\left(x^{2}, n, p^{1}, p^{3}\right)=U_{-1}^{(-p)}\left(x^{2}, n,-p^{1},-p^{3}\right) \text { for } n \geq 0 \\
& \left(C V_{-1}^{(p)}\right)\left(x^{2}, n, p^{1}, p^{3}\right)=-U_{+1}^{(-p)}\left(x^{2}, n,-p^{1},-p^{3}\right) \text { for } n \geq 1
\end{aligned}
$$

The solutions of the right hand side of (2.37) are solutions of the Dirac equation for the positrons and antiprotons with a positive energy.

By (2.37) we set

$$
\begin{aligned}
& U^{(e)}\left(x^{2}, \xi_{1}\right)=U_{s}^{(e)}\left(x^{2}, n, p_{e}^{1}, p_{e}^{3}\right) \text { for } \xi_{1}=\left(s, n, p_{e}^{1}, p_{e}^{3}\right), n \geq 0 \\
& W^{(e)}\left(x^{2}, \xi_{1}\right)=V_{-1}^{(e)}\left(x^{2}, n,-p_{e}^{1},-p_{e}^{3}\right) \text { for } \xi_{1}=\left(1, n, p_{e}^{1}, p_{e}^{3}\right), n \geq 0 \\
& W^{(e)}\left(x^{2}, \xi_{1}\right)=V_{+1}^{(e)}\left(x^{2}, n,-p_{e}^{1},-p_{e}^{3}\right) \text { for } \xi_{1}=\left(-1, n, p_{e}^{1}, p_{e}^{3}\right), n \geq 1 \\
& W^{(e)}\left(x^{2}, \xi_{1}\right)=0 \text { for } \xi_{1}=\left(-1,0, p_{e}^{1}, p_{e}^{3}\right)
\end{aligned}
$$

By using (2.37) and (2.38) the symmetric of charge canonical quantization of the classical field (2.17) gives the following formal operator associated with the electron and denoted by $\Psi_{(e)}(x)$ :

$$
\begin{aligned}
\Psi_{(e)}(\mathbf{x})= & \frac{1}{2 \pi} \int \mathrm{d} \xi_{1}\left(\mathrm{e}^{i\left(p_{e}^{1} x^{1}+p_{e}^{3} x^{3}\right)} U^{(e)}\left(x^{2}, \xi_{1}\right) b_{+}\left(\xi_{1}\right)\right. \\
& \left.+\mathrm{e}^{-i\left(p_{e}^{1} x^{1}+p_{e}^{3} x^{3}\right)} W^{(e)}\left(x^{2}, \xi_{1}\right) b_{-}^{*}\left(\xi_{1}\right)\right)
\end{aligned}
$$

For a rigourous approach of the quantization see [22].

We further note that

$$
\left\{\Psi_{(e)}(\mathbf{x}), \Psi_{(e)}\left(\mathbf{x}^{\prime}\right)^{\dagger}\right\}=\delta\left(\mathbf{x}, \mathbf{x}^{\prime}\right)
$$

See [11]. 
By (2.37) we now set

$$
\begin{aligned}
& U^{(p)}\left(x^{2}, \xi_{2}\right)=U_{s}^{(p)}\left(x^{2}, n, p_{p}^{1}, p_{p}^{3}\right) \text { for } \xi_{2}=\left(s, n,-p_{p}^{1},-p_{p}^{3}\right), n \geq 0 \\
& W^{(p)}\left(x^{2}, \xi_{2}\right)=V_{+1}^{(p)}\left(x^{2}, n,-p_{p}^{1},-p_{p}^{3}\right) \text { for } \xi_{2}=\left(-1, n, p_{p}^{1}, p_{p}^{3}\right), n \geq 0 \\
& W^{(p)}\left(x^{2}, \xi_{2}\right)=V_{-1}^{(p)}\left(x^{2}, n,-p_{p}^{1},-p_{p}^{3}\right) \text { for } \xi_{2}=\left(1, n, p_{p}^{1}, p_{p}^{3}\right), n \geq 1 \\
& W^{(p)}\left(x^{2}, \xi_{2}\right)=0 \quad \text { when } \xi_{2}=\left(1,0, p_{p}^{1}, p_{p}^{3}\right)
\end{aligned}
$$

By using (2.37) and (2.41) the symmetric of charge canonical quantization of the classical field (2.26) gives the following formal operator associated to the proton and denoted by $\Psi_{(p)}(x)$ :

$$
\begin{aligned}
\Psi_{(p)}(\mathbf{x})= & \frac{1}{2 \pi} \int \mathrm{d} \xi_{2}\left(\mathrm{e}^{i\left(p_{p}^{1} x^{1}+p_{p}^{3} x^{3}\right)} U^{(p)}\left(x^{2}, \xi_{2}\right) b_{+}\left(\xi_{2}\right)\right. \\
& \left.+\mathrm{e}^{-i\left(p_{p}^{1} x^{1}+p_{p}^{3} x^{3}\right)} W^{(p)}\left(x^{2}, \xi_{2}\right) b_{-}^{*}\left(\xi_{2}\right)\right)
\end{aligned}
$$

We further note that

$$
\left\{\Psi_{(p)}(\mathbf{x}), \Psi_{(p)}\left(\mathbf{x}^{\prime}\right)^{\dagger}\right\}=\delta\left(\mathbf{x}-\mathbf{x}^{\prime}\right)
$$

See [11].

\section{The Quantization of the Dirac Fields for the Neutrons and the Neutrinos in Helicity Formalism}

As stated in the introduction we neglect the magnetic moment of the neutrons. Therefore neutrons and neutrinos are purely neutral particles without any electromagnetic interaction. We suppose that the neutrinos and antineutrinos are massless as in the Standard Model.

The quantized Dirac fields for free massive and massless particles of $\operatorname{spin} \frac{1}{2}$ are well-known.

In this work we use the helicity formalism, for free particles. See, for example, [7] [15] and [16].

The helicity formalism for particles is associated with a spectral representation of the set of commuting self adjoint operators $\left(\mathbf{P}, \mathrm{H}^{3}\right) . \quad \mathbf{P}=\left(\mathrm{P}^{1}, \mathrm{P}^{2}, \mathrm{P}^{3}\right)$ are the generators of space-translations and $\mathrm{H}^{3}$ is the helicity operator $\frac{1}{2} \frac{\mathbf{P} \cdot \boldsymbol{\Sigma}}{|\mathbf{P}|}$ where $|\mathbf{P}|=\left(\sqrt{\sum_{i=1}^{3}\left(\mathrm{P}^{i}\right)^{2}}\right)$ and $\boldsymbol{\Sigma}=\left(\Sigma^{1}, \Sigma^{2}, \Sigma^{3}\right)$ with for $j=1,2,3$

$$
\Sigma^{j}=\left(\begin{array}{cc}
\sigma_{j} & 0 \\
0 & \sigma_{j}
\end{array}\right)
$$

\subsection{The Quantization of the Dirac Field for the Neutron in Helicity Formalism}

The Dirac equation for the neutron of mass $m_{n}$ is given by

$$
H_{D}=\alpha \cdot \frac{1}{i} \nabla+\beta m_{n}
$$


acting in the Hilbert space $L^{2}\left(\mathbb{R}^{3}, \mathbb{C}^{4}\right)$.

It follows from the Fourier transform that

$$
\begin{aligned}
& L^{2}\left(\mathbb{R}^{3}, \mathbb{C}^{4}\right) \simeq \int_{\mathbb{R}^{3}}^{\oplus} \mathbb{C}^{4} \mathrm{~d}^{3} p . \\
& H_{D} \simeq \int_{\mathbb{R}^{3}}^{\oplus} H_{D}(\mathbf{p}) \mathrm{d}^{3} p
\end{aligned}
$$

where

$$
H_{D}(\mathbf{p})=\left(\begin{array}{cc}
m_{n} \sigma_{0} & \sigma \cdot \mathbf{p} \\
\sigma \cdot \mathbf{p} & -m_{n} \sigma_{0}
\end{array}\right)
$$

Here $\sigma_{0}$ is the $2 \times 2$ unit matrix, $\sigma=\left(\sigma_{1}, \sigma_{2}, \sigma_{3}\right)$ and $\mathbf{p}=\left(p^{1}, p^{2}, p^{3}\right)$ with $\sigma \cdot \mathbf{p}=\sum_{j=1}^{3} \sigma_{j} p^{j}$.

$$
H_{D}(\mathbf{p}) \text { has two eigenvalues } E^{(n)}(\mathbf{p}) \text { and }-E^{(n)}(\mathbf{p}) \text { where }
$$

$$
E^{(n)}(\mathbf{p})=\sqrt{|\mathbf{p}|^{2}+m_{n}^{2}}
$$

The helicity, denoted by $H_{3}(\mathbf{p})$, is given by

$$
H_{3}(\mathbf{p})=\frac{1}{2}\left(\begin{array}{cc}
\frac{\sigma \cdot \mathbf{p}}{|\mathbf{p}|} & 0 \\
0 & \frac{\sigma \cdot \mathbf{p}}{|\mathbf{p}|}
\end{array}\right)
$$

$H_{3}(\mathbf{p})=$ commutes with $H_{D}(\mathbf{p})$ and has two eigenvalues $\frac{1}{2}$ and $-\frac{1}{2}$.

Set (see ([7], Appendix. 1.F.] and [15]) for $|\mathbf{p}| \neq p^{3}$

$$
h_{+}(\mathbf{p})=\frac{1}{\sqrt{2|\mathbf{p}|\left(|\mathbf{p}|-p^{3}\right)}}\left(\begin{array}{l}
p^{1}-i p^{2} \\
|\mathbf{p}|-p^{3}
\end{array}\right)
$$

and

$$
h_{-}(\mathbf{p})=\frac{1}{\sqrt{2|\mathbf{p}|\left(|\mathbf{p}|-p^{3}\right)}}\left(\begin{array}{c}
p^{3}-|\mathbf{p}| \\
p^{1}+i p^{2}
\end{array}\right)
$$

For $|\mathbf{p}|=p^{3}$ we set

$$
h_{+}(\mathbf{p})=\left(\begin{array}{l}
1 \\
0
\end{array}\right)
$$

and

$$
h_{-}(\mathbf{p})=\left(\begin{array}{l}
0 \\
1
\end{array}\right)
$$

We have $(\sigma \cdot \mathbf{p}) h_{ \pm}(\mathbf{p})= \pm|\mathbf{p}| h_{ \pm}(\mathbf{p})$.

Let

$$
a_{ \pm}(\mathbf{p})=\frac{1}{\sqrt{2}}\left(1 \pm \frac{m_{n}}{E^{(n)}(\mathbf{p})}\right)^{\frac{1}{2}}
$$

The two eigenfunctions of the eigenvalue $E^{(n)}(\mathbf{p})$ associated with helicities 


$$
\begin{array}{r}
\frac{1}{2} \text { and }-\frac{1}{2} \text { are denoted by } U^{(n)}\left(\mathbf{p}, \pm \frac{1}{2}\right) \text { and are given by } \\
U^{(n)}\left(\mathbf{p}, \pm \frac{1}{2}\right)=\left(\begin{array}{c}
a_{+}(\mathbf{p}) h_{ \pm}(\mathbf{p}) \\
\pm a_{-}(\mathbf{p}) h_{ \pm}(\mathbf{p})
\end{array}\right)
\end{array}
$$

We now turn to the eigenfunctions for the eigenvalue $-E^{(n)}(\mathbf{p})$.

The two eigenfunctions associated with the eigenvalue $-E^{(n)}(\mathbf{p})$ and with helicities $\frac{1}{2}$ and $-\frac{1}{2}$ are denoted by $V^{(n)}\left(\mathbf{p}, \pm \frac{1}{2}\right)$ and are given by

$$
V^{(n)}\left(\mathbf{p}, \pm \frac{1}{2}\right)=\left(\begin{array}{c}
\mp a_{-}(\mathbf{p}) h_{ \pm}(\mathbf{p}) \\
a_{+}(\mathbf{p}) h_{ \pm}(\mathbf{p})
\end{array}\right)
$$

The four vectors $U^{(n)}\left(\mathbf{p}, \pm \frac{1}{2}\right)$ and $V^{(n)}\left(\mathbf{p}, \pm \frac{1}{2}\right)$ form an orthonormal basis of $\mathbb{C}^{4}$.

$$
U^{(n)}\left(\mathbf{p}, \pm \frac{1}{2}\right) \mathrm{e}^{i(\mathbf{p} \cdot \mathbf{x})} \text { and } V^{(n)}\left(\mathbf{p}, \pm \frac{1}{2}\right) \mathrm{e}^{i(\mathbf{p} \cdot \mathbf{x})} \text { is a complete set of generalized }
$$
eigenfunctions of (3.2) with positive and negative eigenvalues $\pm E^{(n)}(\mathbf{p})$.

This yields for $\Psi(\mathbf{x})$ in $L^{2}\left(\mathbb{R}^{3}, \mathbb{C}^{4}\right)$

$$
\begin{aligned}
& \Psi(\boldsymbol{x}) \\
& =\left(\frac{1}{2 \pi}\right)^{\frac{3}{2}} \sum_{\lambda= \pm \frac{1}{2}} \text { L.i.m. }\left(\int_{\mathbb{R}^{3}} \mathrm{~d}^{3} p \mathrm{e}^{i(\boldsymbol{p} \cdot \boldsymbol{x})}\left(U^{(n)}(\boldsymbol{p}, \lambda) a(\boldsymbol{p}, \lambda)+V^{(n)}(\boldsymbol{p}, \lambda) c(\boldsymbol{p}, \lambda)\right)\right)
\end{aligned}
$$

with

$$
\|\Psi(.)\|_{L^{2}\left(\mathbb{R}^{3}, \mathbb{C}^{4}\right)}^{2}=\sum_{\lambda= \pm \frac{1}{2}} \int_{\mathbb{R}^{3}} \mathrm{~d}^{3} p\left(|a(\mathbf{p}, \lambda)|^{2}+|c(\mathbf{p}, \lambda)|^{2}\right)<\infty
$$

\subsubsection{Fock Space for the Neutrons}

We recall that the neutron is not its own antiparticle.

Let $\xi_{3}=(\mathbf{p}, \lambda)$ be the quantum variables of a neutron and an antineutron where $\mathbf{p} \in \mathbb{R}^{3}$ is the momentum and $\lambda \in\left\{-\frac{1}{2}, \frac{1}{2}\right\}$ is the helicity. We set $\Gamma_{2}=\mathbb{R}^{3} \times\left\{-\frac{1}{2}, \frac{1}{2}\right\}$ for the configuration space of the neutron and the antineutron.

Let $\mathfrak{F}_{(n)}$ and $\mathfrak{F}_{(\bar{n})}$ denote the Fock spaces for the neutrons and the antineutrons respectively.

We have

$$
\mathfrak{F}_{(n)}=\mathfrak{F}_{(\bar{n})}=\bigoplus_{n=0}^{\infty} \bigotimes_{a}^{n} L^{2}\left(\Gamma_{2}\right)
$$

$\bigotimes_{a}^{n} L^{2}\left(\Gamma_{2}\right)$ is the antisymmetric $n$-th tensor power of $L^{2}\left(\Gamma_{2}\right)$.

$\Omega_{(\beta)}=(1,0,0,0, \cdots)$ is the vacuum state in $\mathfrak{F}_{(\beta)}$ for $\beta=n, \bar{n}$.

In the sequel we shall use the notations 


$$
\int_{\Gamma_{2}} \mathrm{~d} \xi_{3}=\sum_{\lambda= \pm \frac{1}{2}} \int_{\mathbb{R}^{3}} \mathrm{~d}^{3} p
$$

$b_{\epsilon}\left(\xi_{3}\right)$ (resp. $b_{\epsilon}^{*}\left(\xi_{3}\right)$ ) is the annihilation (resp.creation)operator for the neutron if $\epsilon=+$ and for the antineutron if $\epsilon=-$.

The operators $b_{\epsilon}\left(\xi_{3}\right)$ and $b_{\epsilon}^{*}\left(\xi_{3}\right)$ fulfil the usual anticommutation relations (CAR) and they anticommute with $b_{\epsilon}^{\#}\left(\xi_{j}\right)$ for $j=1,2$ according to the convention described in ([12], Section 4.1). See [13] arXiv for explicit definitions.

Therefore the following anticommutation relations hold for $j=1,2$

$$
\begin{aligned}
& \left\{b_{\epsilon}\left(\xi_{3}\right), b_{\epsilon^{\prime}}^{*}\left(\xi_{3}^{\prime}\right)\right\}=\delta_{\epsilon \epsilon^{\prime}} \delta\left(\xi_{3}-\xi_{3}^{\prime}\right), \\
& \left\{b_{\epsilon}^{\#}\left(\xi_{3}\right), b_{\epsilon^{\prime}}^{\#}\left(\xi_{j}\right)\right\}=0
\end{aligned}
$$

Recall that for $\varphi \in L^{2}\left(\Gamma_{2}\right)$, the operators

$$
\begin{aligned}
b_{3, \epsilon}(\varphi) & =\int_{\Gamma_{2}} b_{\epsilon}\left(\xi_{3}\right) \overline{\varphi\left(\xi_{3}\right)} \mathrm{d} \xi_{3} . \\
b_{3, \epsilon}^{*}(\varphi) & =\int_{\Gamma_{2}} b_{\epsilon}^{*}\left(\xi_{3}\right) \varphi\left(\xi_{3}\right) \mathrm{d} \xi_{3}
\end{aligned}
$$

are bounded operators on $\mathfrak{F}_{(n)}$ and $\mathfrak{F}_{(\bar{n})}$ satisfying

$$
\left\|b_{3, \epsilon}^{\#}(\varphi)\right\|=\|\varphi\|_{L^{2}}
$$

\subsubsection{Quantized Dirac Field for the Neutron in Helicity Formalism} By (2.35) we get

$$
\begin{aligned}
& C\left(V^{(n)}\left(\mathbf{p}, \frac{1}{2}\right)\right)=\left(-\frac{p^{1}+i p^{2}}{\left|p^{1}+i p^{2}\right|}\right) U^{(n)}\left(-\mathbf{p}, \frac{1}{2}\right) \\
& C\left(V^{(n)}\left(\mathbf{p},-\frac{1}{2}\right)\right)=\left(-\frac{p^{1}-i p^{2}}{\left|p^{1}+i p^{2}\right|}\right) U^{(n)}\left(-\mathbf{p},-\frac{1}{2}\right)
\end{aligned}
$$

Setting

$$
\begin{gathered}
U^{(n)}\left(\xi_{3}\right)=U^{(n)}(\mathbf{p}, \lambda) \\
W^{(n)}\left(\xi_{3}\right)=V^{(n)}(-\mathbf{p}, \lambda)
\end{gathered}
$$

and applying the canonical quantization we obtain the following quantized Dirac field for the neutron:

$$
\Psi_{(n)}(\mathbf{x})=\left(\frac{1}{2 \pi}\right)^{\frac{3}{2}} \int \mathrm{d} \xi_{3}\left(\mathrm{e}^{i(\mathbf{p} \cdot \mathbf{x})} U^{(n)}\left(\xi_{3}\right) b_{+}\left(\xi_{3}\right)+\mathrm{e}^{-i(\mathbf{p} \cdot \mathbf{x})} W^{(n)}\left(\xi_{3}\right) b_{-}^{*}\left(\xi_{3}\right)\right)
$$

\subsection{The Quantization of the Dirac Field for the Neutrino}

Throughout this work we suppose that the neutrinos we consider are those associated with the electrons.

The Dirac equation for the neutrino is given by

$$
H_{D}=\alpha \cdot \frac{1}{i} \nabla
$$

acting in the Hilbert space $L^{2}\left(\mathbb{R}^{3}, \mathbb{C}^{4}\right)$.

By (3.3) it follows from the Fourier transform that 


$$
H_{D} \simeq \int_{\mathbb{R}^{3}}^{\oplus} H_{D}(\mathbf{p}) \mathrm{d}^{3} p
$$

where

$$
H_{D}(\mathbf{p})=\left(\begin{array}{cc}
0 & \sigma \cdot \mathbf{p} \\
\sigma \cdot \mathbf{p} & 0
\end{array}\right)
$$

$H_{D}(\mathbf{p})$ has two eigenvalues $E^{(v)}(\mathbf{p})$ and $-E^{(v)}(\mathbf{p})$ where $E^{(v)}(\mathbf{p})=|\mathbf{p}|$. The helicity given by

$$
\frac{1}{2} \gamma_{5}=\frac{1}{2}\left(\begin{array}{ll}
0 & I \\
I & 0
\end{array}\right)
$$

commutes with $H_{D}(\mathbf{p})$ and has two eigenvalues $\frac{1}{2}$ and $-\frac{1}{2}$.

The two eigenfunctions of the eigenvalue $E^{(v)}(\mathbf{p})$ associated with helicities $\frac{1}{2}$ and $-\frac{1}{2}$ are denoted by $U^{(v)}\left(\mathbf{p}, \pm \frac{1}{2}\right)$. The two eigenfunctions of the eigenvalue $-E^{(v)}(\mathbf{p})$ associated with helicities $\frac{1}{2}$ and $-\frac{1}{2}$ are denoted by $V^{(v)}\left(\mathbf{p}, \pm \frac{1}{2}\right)$. They are given by

$$
\begin{aligned}
& U^{(v)}\left(\mathbf{p}, \pm \frac{1}{2}\right)=\frac{1}{\sqrt{2}}\left(\begin{array}{c}
h_{ \pm}(\mathbf{p}) \\
\pm h_{ \pm}(\mathbf{p})
\end{array}\right) \\
& V^{(v)}\left(\mathbf{p}, \pm \frac{1}{2}\right)=\frac{1}{\sqrt{2}}\left(\begin{array}{c}
\mp h_{ \pm}(\mathbf{p}) \\
h_{ \pm}(\mathbf{p})
\end{array}\right)
\end{aligned}
$$

The four vectors $U^{(v)}\left(\mathbf{p}, \pm \frac{1}{2}\right)$ and $V^{(v)}\left(\mathbf{p}, \pm \frac{1}{2}\right)$ form an orthonormal basis in $\mathbb{C}^{4}$.

Turning now to the theory of neutrinos and antineutrinos (see [17]) a neutrino has a helicity equal to $-\frac{1}{2}$ and a antineutrino a helicity equal to $\frac{1}{2}$. Neutrinos are left-handed and antineutrinos are right-handed. $U^{(v)}\left(\mathbf{p},-\frac{1}{2}\right)$ is the eigenfunction of a neutrino with a momentum $\mathbf{p}$ and an energy $|\mathbf{p}| \cdot C V^{(v)}\left(-\mathbf{p}, \frac{1}{2}\right)$ is the eigenfunction of an antineutrino with a momentum $\mathbf{p}$ and an energy $|\mathbf{p}|$.

Thus the classical field, denoted by $\Phi(\mathbf{x})$ and associated with the neutrino and the antineutrino, is given by

$$
\begin{aligned}
\Phi(\mathbf{x})= & \left(\frac{1}{2 \pi}\right)^{\frac{3}{2}} \text { L.i.m. }\left(\int _ { \mathbb { R } ^ { \mathbb { 2 } } } \mathrm { d } ^ { 3 } p \left(\mathrm{e}^{i(\mathbf{p} \cdot \mathbf{x})} U^{(v)}\left(\mathbf{p},-\frac{1}{2}\right) a\left(\mathbf{p},-\frac{1}{2}\right)\right.\right. \\
& \left.\left.+\mathrm{e}^{-i(\mathbf{p} \cdot \mathbf{x})} V^{(v)}\left(-\mathbf{p}, \frac{1}{2}\right) c\left(\mathbf{p}, \frac{1}{2}\right)\right)\right)
\end{aligned}
$$

with 


$$
\|\Phi(.)\|_{L^{2}\left(\mathbb{R}^{3}, \mathbb{C}^{4}\right)}^{2}=\int_{\mathbb{R}^{3}} \mathrm{~d}^{3} p\left(\left|a\left(\mathbf{p},-\frac{1}{2}\right)\right|^{2}+\left|c\left(\mathbf{p}, \frac{1}{2}\right)\right|^{2}\right)<\infty
$$

\subsubsection{Fock Space for the Neutrinos and the Antineutrinos}

Let $\xi_{4}=\left(\mathbf{p},-\frac{1}{2}\right)$ be the quantum variables of a neutrino where $\mathbf{p} \in \mathbb{R}^{3}$ is the momentum and $-\frac{1}{2}$ is the helicity. In the case of the antineutrino we set $\tilde{\xi}_{4}=\left(\mathbf{p}, \frac{1}{2}\right)$ where $\mathbf{p} \in \mathbb{R}^{3}$ and $\frac{1}{2}$ is the helicity.

$L^{2}\left(\mathbb{R}^{3}\right)$ is the Hilbert space of the states of the neutrinos and of the antineutrinos.

Let $\mathfrak{F}_{(v)}$ and $\mathfrak{F}_{(\bar{v})}$ denote the Fock spaces for the neutrinos and the antineutrinos respectively.

We have

$$
\mathfrak{F}_{(v)}=\mathfrak{F}_{(\bar{v})}=\bigoplus_{n=0}^{\infty} \underset{a}{\otimes} L^{2}\left(\mathbb{R}^{3}\right)
$$

$\bigotimes_{a}^{n} L^{2}\left(\mathbb{R}^{3}\right)$ is the antisymmetric $n$-th tensor power of $L^{2}\left(\mathbb{R}^{3}\right)$.

$\Omega_{(\delta)}=(1,0,0,0, \cdots)$ is the vacuum state in $\mathfrak{F}_{(\delta)}$ for $\delta=v, \bar{v}$.

In the sequel we shall use the notations

$$
\begin{aligned}
\int_{\mathbb{R}^{3}} \mathrm{~d} \xi_{4} & =\int_{\mathbb{R}^{3}} \mathrm{~d}^{3} p \\
\int_{\mathbb{R}^{3}} \mathrm{~d} \tilde{\xi}_{4} & =\int_{\mathbb{R}^{3}} \mathrm{~d}^{3} p
\end{aligned}
$$

$b_{+}\left(\xi_{4}\right)$ (resp. $\left.b_{+}^{*}\left(\xi_{4}\right)\right)$ is the annihilation (resp.creation) operator for the neutrino and $b_{-}\left(\tilde{\xi}_{4}\right)$ (resp. $\left.b_{-}^{*}\left(\tilde{\xi}_{4}\right)\right)$ is the annihilation (resp.creation) operator for the antineutrino.

The operators $b_{+}\left(\xi_{4}\right), b_{+}^{*}\left(\xi_{4}\right), b_{-}\left(\tilde{\xi}_{4}\right)$ and $b_{-}^{*}\left(\tilde{\xi}_{4}\right)$ fulfil the usual anticommutation relations (CAR) and they anticommute with $b_{\epsilon}^{\#}\left(\xi_{j}\right)$ for $j=1,2,3$ according the convention described in ([12], Section 4.1). See [13] arXiv for explicit definitions.

Therefore the following anticommutation relations hold for $j=1,2,3$

$$
\begin{aligned}
& \left\{b_{+}\left(\xi_{4}\right), b_{+}^{*}\left(\xi_{4}^{\prime}\right)\right\}=\delta\left(\xi_{4}-\xi_{4}^{\prime}\right), \\
& \left\{b_{-}\left(\tilde{\xi}_{4}\right), b_{-}^{*}\left(\tilde{\xi}_{4}^{\prime}\right)\right\}=\delta\left(\tilde{\xi}_{4}-\tilde{\xi}_{4}^{\prime}\right), \\
& \left\{b_{+}^{\#}\left(\xi_{4}\right), b_{-}^{\#}\left(\tilde{\xi}_{4}^{\prime}\right)\right\}=0, \\
& \left\{b_{+}^{\#}\left(\xi_{4}\right), b_{\epsilon}^{\#}\left(\xi_{j}\right)\right\}=\left\{b_{-}^{\#}\left(\tilde{\xi}_{4}\right), b_{\epsilon}^{\#}\left(\xi_{j}\right)\right\}=0
\end{aligned}
$$

Recall that for $\varphi \in L^{2}\left(\mathbb{R}^{3}\right)$, the operators

$$
\begin{aligned}
& b_{4,+}(\varphi)=\int_{\mathbb{R}^{3}} b_{+}\left(\xi_{4}\right) \overline{\varphi\left(\xi_{4}\right)} \mathrm{d} \xi_{4} \\
& b_{4,-}(\varphi)=\int_{\mathbb{R}^{3}} b_{-}\left(\tilde{\xi}_{4}\right) \overline{\varphi\left(\tilde{\xi}_{4}\right)} \mathrm{d} \tilde{\xi}_{4} \\
& b_{4,+}^{*}(\varphi)=\int_{\mathbb{R}^{3}} b_{+}^{*}\left(\xi_{4}\right) \varphi\left(\xi_{4}\right) \mathrm{d} \xi_{4} \\
& b_{4,-}^{*}(\varphi)=\int_{\mathbb{R}^{3}} b_{+}^{*}\left(\tilde{\xi}_{4}\right) \varphi\left(\tilde{\xi}_{4}\right) \mathrm{d} \tilde{\xi}_{4}
\end{aligned}
$$


are bounded operators on $\mathfrak{F}_{(v)}$ and $\mathfrak{F}_{(\bar{v})}$ respectively satisfying

$$
\left\|b_{4, \epsilon}^{\#}(\varphi)\right\|=\|\varphi\|_{L^{2}}
$$

where $\epsilon= \pm$.

\subsubsection{Quantized Dirac Field for the Neutrino}

$\mathrm{e}^{i(\mathbf{p} \cdot \mathbf{x})} U^{(v)}\left(\mathbf{p}_{v},-\frac{1}{2}\right)$ and $\mathrm{e}^{i(\mathbf{p} \cdot \mathbf{x})} V^{(v)}\left(\mathbf{p}, \frac{1}{2}\right)$ are generalized eigenfunctions of

with positive and negative eigenvalues $\pm E^{(v)}(\mathbf{p})$ respectively.

By (2.35) we get

$$
C\left(V^{(v)}\left(\mathbf{p}, \frac{1}{2}\right)\right)=\left(-\frac{p^{1}+i p^{2}}{\left|p^{1}+i p^{2}\right|}\right) U^{(v)}\left(-\mathbf{p}, \frac{1}{2}\right)
$$

Setting

$$
\begin{aligned}
& U^{(v)}\left(\mathbf{p},-\frac{1}{2}\right)=U^{(v)}\left(\xi_{4}\right) \\
& V^{(v)}\left(-\mathbf{p}, \frac{1}{2}\right)=W^{(v)}\left(\tilde{\xi}_{4}\right)
\end{aligned}
$$

and applying the canonical quantization we obtain the following quantized Dirac field for the neutrino:

$$
\Psi_{(v)}(\mathbf{x})=\left(\frac{1}{2 \pi}\right)^{\frac{3}{2}}\left(\int \mathrm{d} \xi_{4} \mathrm{e}^{i(\mathbf{p} \cdot \mathbf{x})} U^{(v)}\left(\xi_{4}\right) b_{+}\left(\xi_{4}\right)+\int \mathrm{d} \tilde{\xi}_{4} e^{-i(\mathbf{p} \cdot \mathbf{x})} W^{(v)}\left(\tilde{\xi}_{4}\right) b_{-}^{*}\left(\tilde{\xi}_{4}\right)\right)
$$

\section{The Hamiltonian of the Model}

The processes (1.1) and (1.2) are associated with the $\beta$ decay of the neutron (see [3] [4] [17] and [18]).

The $\beta$ decay process can be described by the well known four-fermion effective Hamiltonian for the interaction in the Schrdinger representation:

$$
\begin{aligned}
H_{\text {int }}= & \frac{\tilde{G}}{\sqrt{2}} \int \mathrm{d}^{3} x\left(\overline{\Psi_{(p)}}(x) \gamma^{\alpha}\left(1-g_{A} \gamma_{5}\right) \Psi_{(n)}(x)\right)\left(\overline{\Psi_{(e)}}(x) \gamma_{\alpha}\left(1-\gamma_{5}\right) \Psi_{(v)}(x)\right) \\
& +\frac{\tilde{G}}{\sqrt{2}} \int \mathrm{d}^{3} x\left(\overline{\Psi_{(v)}}(x) \gamma_{\alpha}\left(1-\gamma_{5}\right) \Psi_{(e)}(x)\right)\left(\overline{\Psi_{(n)}}(x) \gamma^{\alpha}\left(1-g_{A} \gamma_{5}\right) \Psi_{(p)}(x)\right)
\end{aligned}
$$

Here $\gamma^{\alpha}, \alpha=0,1,2,3$ and $\gamma_{5}$ are the Dirac matrices in the standard representation. $\Psi_{(.)}(x)$ and $\overline{\Psi_{(.)}}(x)$ are the quantized Dirac fields for $p, n, e$ and $v . \overline{\Psi_{(.)}}(x)=\Psi_{(.)}(x)^{\dagger} \gamma^{0} . \tilde{G}=G_{F} \cos \theta_{c}$, where $G_{F}$ is the Fermi coupling constant with $G_{F} \simeq 1.16639(2) \times 10^{-5} \mathrm{GeV}^{-2}$ and $\theta_{c}$ is the Cabbibo angle with $\cos \theta_{c} \simeq 0.9751$. Moreover $g_{A} \simeq 1.27$. See [19].

The neutrino $v$ is the neutrino associated to the electron and usually denoted by $v_{e}$ in Physics.

From now on we restrict ourselves to the study of processes (1.1).

We recall that $m_{e}<m_{p}<m_{p}$. 


\subsection{The Free Hamiltonian}

We set

$$
\begin{aligned}
& \mathfrak{F}^{(e)}=\mathfrak{F}_{(e)} \otimes \mathfrak{F}_{(-e)} \cdot \\
& \mathfrak{F}^{(p)}=\mathfrak{F}_{(p)} \otimes \mathfrak{F}_{(-p)} \cdot \\
& \mathfrak{F}^{(n)}=\mathfrak{F}_{(n)} \\
& \mathfrak{F}^{(v)}=\mathfrak{F}_{(v)} \cdot \\
& \mathfrak{F}=\mathfrak{F}^{(e)} \otimes \mathfrak{F}^{(p)} \otimes \mathfrak{F}^{(n)} \otimes \mathfrak{F}^{(v)}
\end{aligned}
$$

We set

$$
\begin{aligned}
& \omega\left(\xi_{1}\right)=E_{n}^{(e)}\left(p^{3}\right) \text { for } \xi_{1}=\left(s, n, p^{1}, p^{3}\right) \\
& \omega\left(\xi_{2}\right)=E_{n}^{(p)}\left(p^{3}\right) \text { for } \xi_{2}=\left(s, n, p^{1}, p^{3}\right) \\
& \omega\left(\xi_{3}\right)=\sqrt{|p|^{2}+m_{n}^{2}} \text { for } \xi_{3}=(p, \lambda) \\
& \omega\left(\xi_{4}\right)=|\mathbf{p}| \text { for } \xi_{4}=\left(p,-\frac{1}{2}\right)
\end{aligned}
$$

Let $H_{D}^{(e)}$ (resp. $H_{D}^{(p)}, H_{D}^{(n)}$ and $H_{D}^{(v)}$ ) be the Dirac Hamiltonian for the electron (resp.the proton, the neutron and the neutrino).

The quantization of $H_{D}^{(e)}$, denoted by $H_{0, D}^{(e)}$ and acting on $\mathfrak{F}^{(e)}$, is given by

$$
H_{0, D}^{(e)}=\sum_{\epsilon= \pm} \int \omega\left(\xi_{1}\right) b_{\epsilon}^{*}\left(\xi_{1}\right) b_{\epsilon}\left(\xi_{1}\right) \mathrm{d} \xi_{1}
$$

Likewise the quantization of $H_{D}^{(p)}, H_{D}^{(n)}$ and $H_{D}^{(v)}$, denoted by $H_{0, D}^{(p)}$, $H_{0, D}^{(n)}$ and $H_{0, D}^{(v)}$ respectively,acting on $\mathfrak{F}^{(p)}, \mathfrak{F}^{(n)}$ and $\mathfrak{F}^{(v)}$ respectively, is given by

$$
\begin{aligned}
& H_{0, D}^{(p)}=\sum_{\epsilon= \pm} \int \omega\left(\xi_{2}\right) b_{\epsilon}^{*}\left(\xi_{2}\right) b_{\epsilon}\left(\xi_{2}\right) \mathrm{d} \xi_{2} \\
& H_{0, D}^{(n)}=\int \omega\left(\xi_{3}\right) b_{+}^{*}\left(\xi_{3}\right) b_{+}\left(\xi_{3}\right) \mathrm{d} \xi_{3} \\
& H_{0, D}^{(v)}=\int \omega\left(\xi_{4}\right) b_{+}^{*}\left(\xi_{4}\right) b_{+}\left(\xi_{4}\right) \mathrm{d} \xi_{4}
\end{aligned}
$$

For each Fock space $\mathfrak{F}^{(\cdot)}$, let $\mathfrak{D}^{(.)}$denote the set of vectors $\Phi \in \mathfrak{F}^{(.)}$for which each component $\Phi^{(r)}$ is smooth and has a compact support and $\Phi^{(r)}=0$ for all but finitely many $(r)$. Then $H_{0, D}^{(.)}$is well-defined on the dense subset $\mathfrak{D}^{(.)}$and it is essentially self-adjoint on $\mathfrak{D}^{(.)}$. The self-adjoint extension will be denoted by the same symbol $H_{0, D}^{(.)}$with domain $D\left(H_{0, D}^{(.)}\right)$.

The spectrum of $H_{0, D}^{(e)} \in \mathfrak{F}^{(e)}$ is given by

$$
\operatorname{spec}\left(H_{0, D}^{(e)}\right)=\{0\} \cup\left[m_{e}, \infty\right)
$$

$\{0\}$ is a simple eigenvalue whose the associated eigenvector is the vacuum in $\mathfrak{F}^{(e)}$ denoted by $\Omega^{(e)} \cdot\left[m_{e}, \infty\right)$ is the absolutely continuous spectrum of $H_{0, D}^{(e)}$.

Likewise the spectra of $H_{0, D}^{(p)}, H_{0, D}^{(n)}$ and $H_{0, D}^{(v)}$ are given by

$$
\begin{aligned}
& \operatorname{spec}\left(H_{0, D}^{(p)}\right)=\{0\} \cup\left[m_{p}, \infty\right) \\
& \operatorname{spec}\left(H_{0, D}^{(n)}\right)=\{0\} \cup\left[m_{n}, \infty\right) \\
& \operatorname{spec}\left(H_{0, D}^{(v)}\right)=[0, \infty)
\end{aligned}
$$

$\Omega^{(p)}, \Omega^{(n)}$ and $\Omega^{(v)}$ are the associated vacua in $\mathfrak{F}^{(p)}, \mathfrak{F}^{(n)}$ and $\mathfrak{F}^{(v)}$ 
respectively and are the associated eigenvectors of $H_{0, D}^{(p)}, H_{0, D}^{(n e)}$ and $H_{0, D}^{(v)}$ respectively for the eigenvalue $\{0\}$.

The vacuum in $\mathfrak{F}$, denoted by $\Omega$, is then given by

$$
\Omega=\Omega^{(e)} \otimes \Omega^{(p)} \otimes \Omega^{(n)} \otimes \Omega^{(v)}
$$

The free Hamiltonian for the model, denoted by $H_{0}$ and acting on $\mathfrak{F}$, is now given by

$$
H_{0}=H_{0, D}^{(e)} \otimes \mathbb{1} \otimes \mathbb{1} \otimes \mathbb{1}+\mathbb{1} \otimes H_{0, D}^{(p)} \otimes \mathbb{1} \otimes \mathbb{1}+\mathbb{1} \otimes \mathbb{1} \otimes H_{0, D}^{(n)} \otimes \mathbb{1}+\mathbb{1} \otimes \mathbb{1} \otimes \mathbb{1} \otimes H_{0, D}^{(v)}
$$

$H_{0}$ is essentially self-adjoint on $\mathfrak{D}=\mathfrak{D}^{(e)} \hat{\otimes} \mathfrak{D}^{(p)} \hat{\otimes} \mathfrak{D}^{(n)} \hat{\otimes} \mathfrak{D}^{(v)}$.

Here $\hat{\otimes}$ is the algebraic tensor product.

$\operatorname{spec}\left(H_{0}\right)=[0, \infty)$ and $\Omega$ is the eigenvector associated with the simple eigenvalue $\{0\}$ of $H_{0}$.

Let $S^{(e)}$ be the set of the thresholds of $H_{0, D}^{(e)}$ :

$$
S^{(e)}=\left(s_{n}^{(e)} ; n \in \mathbb{N}\right)
$$

with $s_{n}^{(e)}=\sqrt{m_{e}^{2}+2 n e B}$.

Likewise let $S^{(p)}$ be the set of the thresholds of $H_{0, D}^{(p)}$ :

$$
S^{(p)}=\left(s_{n}^{(p)} ; n \in \mathbb{N}\right)
$$

with $s_{n}^{(p)}=\sqrt{m_{p}^{2}+2 n e B}$.

Let $S^{(n)}$ be the set of the thresholds of $H_{0, D}^{(n)}$ :

$$
S^{(n)}=\left(n m_{n} ; n \in \mathbb{N} \text {, such that } n \geq 1\right)
$$

Then

$$
\mathfrak{S}=S^{(e)} \cup S^{(p)} \cup S^{(n)}
$$

is the set of the thresholds of $H_{0}$.

\subsection{The Interaction}

By (4.1) let us now write down the formal interaction,denoted by $V_{I}$, involving the protons, the neutrons, the electrons and the neutrinos together with antiparticles in the Schrödinger representation for the process (1.1). We have

$$
V_{I}=V_{I}^{(1)}+V_{I}^{(2)}+V_{I}^{(3)}+V_{I}^{(4)}
$$

Set

$$
\begin{aligned}
& \mathbf{q}=\mathbf{p}_{e}+\mathbf{p}_{p} \\
& \mathbf{r}=\mathbf{p}_{n}+\mathbf{p}_{v}
\end{aligned}
$$

After the integration with respect to $\left(x^{1}, x^{3}\right) \quad V_{I}$ is given by

$$
\begin{aligned}
V_{I}^{(1)}= & \int \mathrm{d} x^{2} \int \mathrm{d} \xi_{1} \mathrm{~d} \xi_{2} \mathrm{~d} \xi_{3} \mathrm{~d} \xi_{4} \mathrm{e}^{i x^{2} r^{2}}\left(\overline{U^{(p)}}\left(x^{2}, \xi_{2}\right) \gamma^{\alpha}\left(1-g_{A} \gamma_{5}\right) U^{(n)}\left(\xi_{3}\right)\right) \\
& \times\left(\overline{U^{(e)}}\left(x^{2}, \xi_{1}\right) \gamma_{\alpha}\left(1-\gamma_{5}\right) U^{(v)}\left(\xi_{4}\right)\right) \\
& \times \delta\left(q^{1}-r^{1}\right) \delta\left(q^{3}-r^{3}\right) b_{+}^{*}\left(\xi_{1}\right) b_{+}^{*}\left(\xi_{2}\right) b_{+}\left(\xi_{3}\right) b_{+}\left(\xi_{4}\right)
\end{aligned}
$$




$$
\begin{aligned}
V_{I}^{(2)}= & \int \mathrm{d} x^{2} \int \mathrm{d} \xi_{1} \mathrm{~d} \xi_{2} \mathrm{~d} \xi_{3} \mathrm{~d} \xi_{4} \mathrm{e}^{-i x^{2} r^{2}}\left(\overline{U^{(v)}}\left(\xi_{4}\right) \gamma_{\alpha}\left(1-\gamma_{5}\right) U^{(e)}\left(x^{2}, \xi_{1}\right)\right) \\
& \times\left(\overline{U^{(n)}}\left(\xi_{3}\right) \gamma^{\alpha}\left(1-g_{A} \gamma_{5}\right) U^{(p)}\left(x^{2}, \xi_{2}\right)\right) \\
& \times \delta\left(q^{1}-r^{1}\right) \delta\left(q^{3}-r^{3}\right) b_{+}^{*}\left(\xi_{4}\right) b_{+}^{*}\left(\xi_{3}\right) b_{+}\left(\xi_{2}\right) b_{+}\left(\xi_{1}\right) \\
V_{I}^{(3)}= & \left.\int \mathrm{d} x^{2} \int \mathrm{d} \xi_{1} \mathrm{~d} \xi_{2} \mathrm{~d} \xi_{3} \mathrm{~d} \xi_{4} \mathrm{e}^{-i x^{2} r^{2}} \overline{U^{(v)}}\left(\xi_{4}\right) \gamma_{\alpha}\left(1-\gamma_{5}\right) W^{(e)}\left(x^{2}, \xi_{1}\right)\right) \\
& \times\left(\overline{U^{(n)}}\left(\xi_{3}\right) \gamma^{\alpha}\left(1-g_{A} \gamma_{5}\right) W^{(p)}\left(x^{2}, \xi_{2}\right)\right) \\
& \times \delta\left(q^{1}+r^{1}\right) \delta\left(q^{3}+r^{3}\right) b_{+}^{*}\left(\xi_{4}\right) b_{+}^{*}\left(\xi_{3}\right) b_{-}^{*}\left(\xi_{2}\right) b_{-}^{*}\left(\xi_{1}\right) \\
V_{I}^{(4)}= & \int \mathrm{d} x^{2} \int \mathrm{d} \xi_{1} \mathrm{~d} \xi_{2} \mathrm{~d} \xi_{3} \mathrm{~d} \xi_{4} \mathrm{e}^{i x^{2} r^{2}}\left(\overline{W^{(p)}}\left(x^{2}, \xi_{2}\right) \gamma^{\alpha}\left(1-g_{A} \gamma_{5}\right) U^{(n)}\left(\xi_{3}\right)\right) \\
& \times\left(\overline{W^{(e)}}\left(x^{2}, \xi_{1}\right) \gamma_{\alpha}\left(1-\gamma_{5}\right) U^{(v)}\left(\xi_{2}\right)\right) \\
& \times \delta\left(q^{1}+r^{1}\right) \delta\left(q^{3}+r^{3}\right) b_{+}\left(\xi_{4}\right) b_{+}\left(\xi_{3}\right) b_{-}\left(\xi_{2}\right) b_{-}\left(\xi_{1}\right)
\end{aligned}
$$

$V_{I}^{(3)}$ and $V_{I}^{(4)}$ are responsible for the fact that the bare vacuum will not be an eigenvector of the total Hamiltonian as expected in Physics.

$V_{I}$ is formally symmetric.

In the Fock space $\mathfrak{F}$ the interaction $V_{I}$ is a highly singular operator due to the $\delta$-distributions that occur in the $\left(V_{I}^{(\cdot)}\right)$ 's and because of the ultraviolet behaviour of the functions $U^{(\cdot)}$ and $W^{(\cdot)}$.

In order to get well defined operators in $\mathfrak{F}$ we have to substitute smoother kernels $F^{(\beta)}\left(\xi_{2}, \xi_{3}\right), G^{(\beta)}\left(\xi_{1}, \xi_{4}\right)$, where $\beta=1,2$, both for the $\delta$-distributions and the ultraviolet cutoffs.

We then obtain a new operator denoted by $H_{I}$ and defined as follows in the Schrödinger representation.

$$
H_{I}=H_{I}^{(1)}+H_{I}^{(2)}+H_{I}^{(3)}+H_{I}^{(4)}
$$

with

$$
\begin{aligned}
H_{I}^{(1)}= & \int \mathrm{d} \xi_{1} \mathrm{~d} \xi_{2} \mathrm{~d} \xi_{3} \mathrm{~d} \xi_{4}\left(\int \mathrm{d} x^{2} \mathrm{e}^{i x^{2} r^{2}}\left(\overline{U^{(p)}}\left(x^{2}, \xi_{2}\right) \gamma^{\alpha}\left(1-g_{A} \gamma_{5}\right) U^{(n)}\left(\xi_{3}\right)\right)\right. \\
& \left.\times\left(\overline{U^{(e)}}\left(x^{2}, \xi_{1}\right) \gamma_{\alpha}\left(1-\gamma_{5}\right) U^{(v)}\left(\xi_{4}\right)\right)\right) \\
& \times F^{(1)}\left(\xi_{2}, \xi_{3}\right) G^{(1)}\left(\xi_{1}, \xi_{4}\right) b_{+}^{*}\left(\xi_{1}\right) b_{+}^{*}\left(\xi_{2}\right) b_{+}\left(\xi_{3}\right) b_{+}\left(\xi_{4}\right) \\
H_{I}^{(2)}= & \int \mathrm{d} \xi_{1} \mathrm{~d} \xi_{2} \mathrm{~d} \xi_{3} \mathrm{~d} \xi_{4}\left(\int \mathrm{d} x^{2} \mathrm{e}^{-i x^{2} r^{2}} \overline{U^{(v)}}\left(\xi_{4}\right) \gamma_{\alpha}\left(1-\gamma_{5}\right) U^{(e)}\left(x^{2}, \xi_{1}\right)\right) \\
& \times\left(\overline{U^{(n)}}\left(\xi_{3}\right) \gamma^{\alpha}\left(1-g_{A} \gamma_{5}\right) U^{(p)}\left(x^{2}, \xi_{2}\right)\right) \\
& \times \overline{F^{(1)}\left(\xi_{2}, \xi_{3}\right)} \overline{G^{(1)}\left(\xi_{1}, \xi_{4}\right)} b_{+}^{*}\left(\xi_{4}\right) b_{+}^{*}\left(\xi_{3}\right) b_{+}\left(\xi_{2}\right) b_{+}\left(\xi_{1}\right) \\
H_{I}^{(3)}= & \int \mathrm{d} \xi_{1} \mathrm{~d} \xi_{2} \mathrm{~d} \xi_{3} \mathrm{~d} \xi_{4}\left(\int \mathrm{d} x^{2} \mathrm{e}^{-i x^{2} r^{2}}\left(\overline{U^{(v)}}\left(\xi_{4}\right) \gamma_{\alpha}\left(1-\gamma_{5}\right) W^{(e)}\left(x^{2}, \xi_{1}\right)\right)\right. \\
& \left.\times\left(\overline{U^{(n)}}\left(\xi_{3}\right) \gamma^{\alpha}\left(1-g_{A} \gamma_{5}\right) W^{(p)}\left(x^{2}, \xi_{2}\right)\right)\right) \\
& \times F^{(2)}\left(\xi_{2}, \xi_{3}\right) G^{(2)}\left(\xi_{1}, \xi_{4}\right) b_{+}^{*}\left(\xi_{4}\right) b_{+}^{*}\left(\xi_{3}\right) b_{-}^{*}\left(\xi_{2}\right) b_{-}^{*}\left(\xi_{1}\right)
\end{aligned}
$$




$$
\begin{aligned}
H_{I}^{(4)}= & \int \mathrm{d} \xi_{1} \mathrm{~d} \xi_{2} \mathrm{~d} \xi_{3} \mathrm{~d} \xi_{4}\left(\int \mathrm{d} x^{2} \mathrm{e}^{i x^{2} r^{2}}\left(\overline{W^{(p)}}\left(x^{2}, \xi_{2}\right) \gamma^{\alpha}\left(1-g_{A} \gamma_{5}\right) U^{(n)}\left(\xi_{3}\right)\right)\right. \\
& \left.\times\left(\overline{W^{(e)}}\left(x^{2}, \xi_{1}\right) \gamma_{\alpha}\left(1-\gamma_{5}\right) U^{(v)}\left(\xi_{4}\right)\right)\right) \\
& \times \overline{F^{(2)}\left(\xi_{2}, \xi_{3}\right) G^{(2)}\left(\xi_{1}, \xi_{4}\right)} b_{+}\left(\xi_{4}\right) b_{+}\left(\xi_{3}\right) b_{-}\left(\xi_{2}\right) b_{-}\left(\xi_{1}\right)
\end{aligned}
$$

Definition 4.1. The total Hamiltonian is

$$
H=H_{0}+g H_{I}
$$

where $g$ is a non-negative coupling constant.

The assumption that $\mathrm{g}$ is non-negative is made for simplicity but all the results below hold for $|g| \in \mathbb{R}$ with $|g|$ small enough.

We now give the hypothesis that the kernels $F^{\beta}(.,),. G^{(\beta)}(.,),. \quad \beta=1,2$, and the coupling constant $g$ have to satisfy in order to associate with the formal operator $H$ a well defined self-adjoint operator in $\mathfrak{F}$.

Throughout this work we assume the following hypothesis

Hypothesis 4.2. For $\beta=1,2$ we assume

$$
\begin{aligned}
& F^{(\beta)}\left(\xi_{2}, \xi_{3}\right) \in L^{2}\left(\Gamma_{1} \times \Gamma_{2}\right) \\
& G^{(\beta)}\left(\xi_{1}, \xi_{4}\right) \in L^{2}\left(\Gamma_{1} \times \mathbb{R}^{3}\right)
\end{aligned}
$$

Let $\langle., .\rangle_{\mathbb{C}^{4}}$ be the scalar product in $\mathbb{C}^{4}$. We have

$$
\begin{aligned}
& \overline{U^{(p)}}\left(x^{2}, \xi_{2}\right) \gamma^{\alpha}\left(1-g_{A} \gamma_{5}\right) U^{(n)}\left(\xi_{3}\right)=\left\langle U^{(p)}\left(x^{2}, \xi_{2}\right), \gamma^{0} \gamma^{\alpha}\left(1-g_{A} \gamma_{5}\right) U^{(n)}\left(\xi_{3}\right)\right\rangle_{\mathbb{C}^{4}} \\
& \overline{U^{(e)}}\left(x^{2}, \xi_{1}\right) \gamma_{\alpha}\left(1-\gamma_{5}\right) U^{(v)}\left(\xi_{4}\right)=\left\langle U^{(e)}\left(x^{2}, \xi_{1}\right), \gamma^{0} \gamma_{\alpha}\left(1-\gamma_{5}\right) U^{(\nu)}\left(\xi_{4}\right)\right\rangle_{\mathbb{C}^{4}} \\
& \overline{U^{(v)}}\left(\xi_{4}\right) \gamma_{\alpha}\left(1-\gamma_{5}\right) W^{(e)}\left(x^{2}, \xi_{1}\right)=\left\langle U^{(\nu)}\left(\xi_{4}\right), \gamma^{0} \gamma_{\alpha}\left(1-\gamma_{5}\right) W^{(e)}\left(x^{2}, \xi_{1}\right)\right\rangle_{\mathbb{C}^{4}} \\
& \overline{U^{(n)}}\left(\xi_{3}\right) \gamma^{\alpha}\left(1-g_{A} \gamma_{5}\right) W^{(p)}\left(x^{2}, \xi_{2}\right)=\left\langle U^{(n)}\left(\xi_{3}\right), \gamma^{0} \gamma^{\alpha}\left(1-g_{A} \gamma_{5}\right) W^{(p)}\left(x^{2}, \xi_{2}\right)\right\rangle_{\mathbb{C}^{4}}
\end{aligned}
$$

Set

$$
C_{0}=\frac{1}{2}\left(\frac{1}{m_{e}}+\frac{1}{m_{n}}\right)\left(\left\|\gamma^{\alpha}\left(1-g_{A} \gamma_{5}\right)\right\|\right)\left(\left\|\gamma_{\alpha}\left(1-\gamma_{5}\right)\right\|\right)
$$

We then have

Proposition 4.3. For every $\Phi \in D\left(H_{0}\right)$ we obtain

$$
\begin{aligned}
& \left\|H_{I}^{(j)} \Phi\right\| \leq C_{0}\left\|F^{(1)}(., .)\right\|_{L^{2}}\left\|G^{(1)}(., .)\right\|_{L^{2}}\left\|\left(H_{0}+m_{n}\right) \Phi\right\| \text { for } j=1,2 \\
& \left\|H_{I}^{(j)} \Phi\right\| \leq C_{0}\left\|F^{(2)}(., .)\right\|_{L^{2}}\left\|G^{(2)}(. . .)\right\|_{L^{2}}\left\|\left(H_{0}+m_{n}\right) \Phi\right\| \text { for } j=3,4
\end{aligned}
$$

By (4.23), (4.24) and (4.25) the estimates (4.26) are examples of $N_{\tau}$ estimates (see [20]). The proof is similar to the one of ([21], Proposition 3.7) and details are omitted.

Let $g_{0}>0$ be such that

$$
2 g_{0} C_{0}\left(\sum_{\beta=1}^{2}\left\|F^{(\beta)}(., .)\right\|_{L^{2}}\left\|G^{(\beta)}(., .)\right\|_{L^{2}}\right)<1
$$

We now have 
Theorem 4.4. For any $g$ such that $g \leq g_{0}, H$ is a self-adjoint operator in $\mathfrak{F}$ with domain $D(H)=D\left(H_{0}\right)$ and is bounded from below. $H$ is essentially self-adjoint on any core of $H_{0}$. Setting

$$
E=\inf \sigma(H)
$$

we have for every $g \leq g_{0}$

$$
\sigma(H)=\sigma_{\text {ess }}(H)=[E, \infty)
$$

with $E \leq 0$.

Here $\sigma(H)$ is the spectrum of $H$ and $\sigma_{\text {ess }}(H)$ is the essential spectrum of $H$.

Proof. By Proposition 4.2 and (4.27) the proof of the self-adjointness of $H$ follows from the Kato-Rellich theorem.

We turn now to the essential spectrum. The result about the essential spectrum in the case of models involving bosons has been obtained by ([14], theorem 4.1) and [23]. In the case of models involving fermions the result has been obtained by [24]. In our case involving only massive fermions and massless neutrinos we use the proof given by [24].

Thus we have to construct a Weyl sequence for $H$ and $E+\lambda$ with $\lambda>0$.

Let $T$ be the self-adjoint multiplication operator in $L^{2}\left(\mathbb{R}^{3}\right)$ defined by $T u\left(\mathbf{p}_{4}\right)=\left|\mathbf{p}_{4}\right| u\left(\mathbf{p}_{4}\right) . T$ is the spectral representation of $H_{D}^{(v)}$ for the neutrinos of helicity $-\frac{1}{2}$ in the configuration space $L^{2}\left(\mathbb{R}^{3}\right)$. See (3.27).

Every $\lambda>0$ belongs to the essential spectrum of $T$. Then there exists a Weyl sequence $\left(f_{n}\right)_{n \geq 1}$ for $T$ and $\lambda>0$ such that

$$
\begin{aligned}
& f_{n} \in D(T) \text { for } n \geq 1 . \\
& \left\|f_{n}\right\|=1 \text { for } n \geq 1 . \\
& w-\lim _{n \rightarrow \infty} f_{n}=0 . \\
& \lim _{n \rightarrow \infty}(T-\lambda) f_{n}=0
\end{aligned}
$$

Let

$$
\begin{aligned}
& f_{n}\left(\xi_{4}\right)=f_{n}\left(\mathbf{p}_{4}\right) \\
& b_{+, 4}\left(f_{n}\right)=\int b_{+}\left(\xi_{4}\right) \overline{f_{n}\left(\xi_{4}\right)} \mathrm{d} \xi_{4} \\
& b_{+, 4}^{*}\left(f_{n}\right)=\int b_{+}^{*}\left(\xi_{4}\right) f_{n}\left(\xi_{4}\right) \mathrm{d} \xi_{4}
\end{aligned}
$$

In the following we identify $b_{+, 4}^{\#}\left(f_{n}\right)$ with its obvious extension to $\mathfrak{F}$. An easy computation shows that, for every $\Psi \in D(H)$,

$$
\begin{aligned}
& {\left[H_{I}^{(1)}, b_{+, 4}^{*}\left(f_{n}\right)\right] \Psi=\int \mathrm{d} \xi_{1} \mathrm{~d} \xi_{2} \mathrm{~d} \xi_{3}\left(\int \mathrm{d} x^{2} \mathrm{e}^{-i x^{2} r^{2}}\right.} \\
& \times\left(\overline{U^{(p)}}\left(x^{2}, \xi_{2}\right) \gamma_{\alpha}\left(1-g_{A} \gamma_{5}\right) U^{(n e)}\left(\xi_{3}\right)\right) F^{(1)}\left(\xi_{2}, \xi_{3}\right) \\
& \left.\times\left\langle U^{(e)}\left(x^{2}, \xi_{1}\right), \gamma^{0} \gamma^{\alpha}\left(1-\gamma_{5}\right)\left(\int f_{n}\left(\xi_{4}\right) G^{(1)}\left(\xi_{1}, \xi_{4}\right) U^{\left(v_{e}\right)}\left(\xi_{4}\right) \mathrm{d} \xi_{4}\right)\right\rangle_{\mathbb{C}^{4}}\right) \\
& \times b_{+}^{*}\left(\xi_{1}\right) b_{+}^{*}\left(\xi_{2}\right) b_{+}\left(\xi_{3}\right) \Psi \\
& {\left[H_{I}^{(1)}, b_{+, 4}\left(f_{n}\right)\right] \Psi=0}
\end{aligned}
$$




$$
\begin{aligned}
& {\left[H_{I}^{(2)}, b_{+, 4}\left(f_{n}\right)\right] \Psi=-\int \mathrm{d} \xi_{1} \mathrm{~d} \xi_{2} \mathrm{~d} \xi_{3}\left(\int \mathrm{d} x^{2} \mathrm{e}^{-i x^{2} r^{2}}\right.} \\
& \times\left(\overline{U^{(n e)}}\left(\xi_{3}\right) \gamma^{\alpha}\left(1-g_{A} \gamma_{5}\right) U^{(p)}\left(x^{2}, \xi_{2}\right)\right) \overline{F^{(1)}\left(\xi_{2}, \xi_{3}\right)} \\
& \left.\times\left\langle\int f_{n}\left(\xi_{4}\right) G^{(1)}\left(\xi_{1}, \xi_{4}\right) U^{(v)}\left(\xi_{4}\right) \mathrm{d} \xi_{4}, \gamma^{0} \gamma^{\alpha}\left(1-\gamma_{5}\right) U^{(e)}\left(x^{2}, \xi_{1}\right)\right\rangle_{\mathbb{C}^{4}}\right) \\
& \times b_{+}^{*}\left(\xi_{3}\right) b_{+}\left(\xi_{2}\right) b_{+}\left(\xi_{1}\right) \Psi \\
& \quad\left[H_{I}^{(2)}, b_{+, 4}^{*}\left(f_{n}\right)\right] \Psi=0 \\
& \quad \times\left(\overline{U^{(n e)}}\left(\xi_{3}\right) \gamma^{\alpha}\left(1-g_{A} \gamma_{5}\right) W^{(p)}\left(x^{2}, \xi_{2}\right)\right) F^{(2)}\left(\xi_{2}, \xi_{3}\right) \\
& \left.\times\left\langle\int f_{n}\left(\xi_{4}\right) \overline{G^{(2)}\left(\xi_{1}, \xi_{4}\right)} U^{(v)}\left(\xi_{4}\right) \mathrm{d} \xi_{4}, \gamma_{0} \gamma_{\alpha}\left(1-\gamma_{5}\right) W^{(e)}\left(x^{2}, \xi_{1}\right)\right\rangle_{\mathbb{C}^{4}}\right) \\
& \times b_{+}^{*}\left(\xi_{3}\right) b_{-}^{*}\left(\xi_{2}\right) b_{-}^{*}\left(\xi_{1}\right) \Psi \\
& {\left[H_{I}^{(4)}, b_{+, 4}^{*}\left(f_{n}\right)\right] \Psi=\int \mathrm{d} \xi_{1} \mathrm{~d} \xi_{2} \mathrm{~d} \xi_{3}\left(\int \mathrm{d} x^{2} \mathrm{e}^{-i x^{2} r^{2}}\right.} \\
& \times\left(\overline{W^{(p)}}\left(x^{2}, \xi_{2}\right) \gamma_{\alpha}\left(1-g_{A} \gamma_{5}\right) U^{(n e)}\left(\xi_{3}\right)\right) \overline{F^{(2)}\left(\xi_{2}, \xi_{3}\right)} \\
& \left.\times\left\langle W^{(e)}\left(x^{2}, \xi_{1}\right), \gamma^{0} \gamma^{\alpha}\left(1-\gamma_{5}\right)\left(\int f_{n}\left(\xi_{4}\right) \overline{G^{(2)}\left(\xi_{1}, \xi_{4}\right)} U^{(v)}\left(\xi_{4}\right) \mathrm{d} \xi_{4}\right)\right\rangle_{\mathbb{C}^{4}}\right) \\
& \times b_{+}\left(\xi_{3}\right) b_{-}\left(\xi_{2}\right) b_{-}\left(\xi_{1}\right) \Psi \\
& {\left[H_{I}^{(4)}, b_{+, 4}\left(f_{n}\right)\right] \Psi=0}
\end{aligned}
$$

Let $P_{H}($.$) be the spectral measure of H$. For any $\epsilon>0$ the orthogonal projection $P_{H}([E, E+\epsilon))$ is different from zero because $E$ belongs to $\sigma(H)$. Let $\Phi_{\varepsilon} \in \operatorname{Ran}\left(P_{H}([E, E+\epsilon))\right)$ such that $\left\|\Phi_{\epsilon}\right\|=1$. We set

$$
\Psi_{n, \epsilon}=\left(b_{+, 4}\left(f_{n}\right)+b_{+, 4}^{*}\left(f_{n}\right)\right) \Phi_{\epsilon}, n \geq 1
$$

Let us chow that there exists a subsequence of $\left(\Psi_{n, \epsilon}\right)_{n \geq 1, \epsilon>0}$ which is a Weyl sequence for $H$ and $E+\lambda$ with $\lambda>0$.

By Hypothesis 4.1, (4.30), (4.32), (4.34), (4.36) and the $N_{\tau}$ estimates we get

$$
\begin{aligned}
& \sup \left(\left\|\left[H_{I}^{(1)}, b_{+, 4}^{*}\left(f_{n}\right)\right] \Psi\right\|,\left\|\left[H_{I}^{(2)}, b_{+, 4}\left(f_{n}\right)\right] \Psi\right\|\right) \\
& \leq C_{0}\left\|F^{(1)}(., .)\right\|_{L^{2}\left(\Gamma_{1} \times \Gamma_{2}\right)}\left(\int\left\|\int f_{n}\left(\xi_{4}\right) G^{(1)}\left(\xi_{1}, \xi_{4}\right) U^{(v)}\left(\xi_{4}\right) \mathrm{d} \xi_{4}\right\|_{\mathbb{C}^{4}}^{2} \mathrm{~d} \xi_{1}\right)^{\frac{1}{2}} \\
& \times\left\|\left(H_{0}+m_{p}\right)^{\frac{1}{2}} \Psi\right\| \\
& \sup \left(\left\|\left[H_{I}^{(3)}, b_{+, 4}\left(f_{n}\right)\right] \Psi\right\|,\left\|\left[H_{I}^{(4)}, b_{+, 4}^{*}\left(f_{n}\right)\right] \Psi\right\|\right) \\
& \leq C_{0}\left\|F^{(2)}(., .)\right\|_{L^{2}\left(\Gamma_{1} \times \Gamma_{2}\right)}\left(\int\left\|\int f_{n}\left(\xi_{4}\right) \overline{G^{(2)}\left(\xi_{1}, \xi_{4}\right)} U^{(v)}\left(\xi_{4}\right) \mathrm{d} \xi_{4}\right\|_{\mathbb{C}^{4}}^{2} \mathrm{~d} \xi_{1}\right)^{\frac{1}{2}} \\
& \quad \times\left\|\left(H_{0}+m_{p}\right)^{\frac{1}{2}} \Psi\right\|
\end{aligned}
$$


Note that

$$
\left\|\Psi_{n, \epsilon}\right\|=1, n \geq 1
$$

We have for every $\Psi \in D(H)$

$$
\begin{aligned}
\left(H \Psi, \Psi_{n, \epsilon}\right)= & \left(\Psi,\left(b_{+, 4}\left(f_{n}\right)+b_{+, 4}^{*}\left(f_{n}\right)\right) H \Phi_{\epsilon}+\left(b_{+, 4}^{*}\left(T f_{n}\right)-b_{+, 4}\left(T f_{n}\right)\right) \Phi_{\epsilon}\right. \\
& \left.+g\left[H_{I},\left(b_{+, 4}\left(f_{n}\right)+b_{+, 4}^{*}\left(f_{n}\right)\right)\right] \Psi_{\epsilon}\right)
\end{aligned}
$$

See [14].

This yields

$$
\begin{aligned}
H \Psi_{n, \epsilon}= & \left(\left(b_{+, 4}\left(f_{n}\right)+b_{+, 4}^{*}\left(f_{n}\right)\right) H \Phi_{\epsilon}+\left(b_{+, 4}^{*}\left(T f_{n}\right)-\left(b_{+, 4}\left(T f_{n}\right)\right) \Phi_{\epsilon}\right.\right. \\
& \left.+g\left[H_{I},\left(b_{+, 4}\left(f_{n}\right)+b_{+, 4}^{*}\left(f_{n}\right)\right)\right] \Psi_{\epsilon}\right)
\end{aligned}
$$

and

$$
\begin{aligned}
(H-E-\lambda) \Psi_{n, \epsilon}= & \left(b_{+, 4}\left(f_{n}\right)+b_{+, 4}^{*}\left(f_{n}\right)\right)(H-E) \Psi_{\epsilon} \\
& +\left(b_{+, 4}\left((T+\lambda) f_{n}\right)+b_{+, 4}^{*}\left((T-\lambda) f_{n}\right)\right) \Psi_{\epsilon} \\
& +g\left[H_{I},\left(b_{+, 4}\left(f_{n}\right)+b_{+, 4}^{*}\left(f_{n}\right)\right)\right] \Psi_{\epsilon}
\end{aligned}
$$

By (3.19) this yields for $g \leq g_{0}$

$$
\begin{aligned}
\left\|(H-E-\lambda) \Psi_{n, \epsilon}\right\| \leq & +2 \epsilon+2|\lambda|\left\|b_{+, 4}\left(f_{n}\right) \Psi_{\epsilon}\right\|+2\left\|\left((T-\lambda) f_{n}\right)\right\| \\
& +g\left\|\left[H_{I}, b_{+, 4}\left(f_{n}\right)\right] \Psi_{\epsilon}\right\|+g\left\|\left[H_{I}, b_{+, 4}^{*}\left(f_{n}\right)\right] \Psi_{\epsilon}\right\|
\end{aligned}
$$

Let $\left\{g_{k} \mid k=1,2,3, \cdots\right\}$ be an orthonormal basis of $L^{2}\left(\mathbb{R}^{3}\right)$ and consider

$$
b_{+, 4}^{*}\left(g_{k_{1}}\right) b_{+, 4}^{*}\left(g_{k_{2}}\right) b_{+, 4}^{*}\left(g_{k_{3}}\right) \cdots b_{+, 4}^{*}\left(g_{k_{m}}\right) \Omega_{v} \in \mathfrak{F}_{(v)}
$$

where the indices can be assumed ordered $k_{1}<\cdots<k_{m}$. Fock space vectors of this type form a basis of $\mathfrak{F}_{(v)}$ (see [7]). By ([24], Lemma 2.1) this yields for every $\epsilon>0$

$$
\begin{aligned}
& s-\lim _{n \rightarrow \infty} b_{+, 4}\left(f_{n}\right) \Psi_{\epsilon}=0, \\
& w-\lim _{n \rightarrow \infty} b_{+, 4}^{*}\left(f_{n}\right) \Psi_{\epsilon}=0
\end{aligned}
$$

By (3.26) and Hypothesis 4.1 we have

$$
\begin{aligned}
& \lim _{n \rightarrow \infty}\left(\int\left\|\int f_{n}\left(\xi_{4}\right) G^{(1)}\left(\xi_{1}, \xi_{4}\right) U^{(v)}\left(\xi_{4}\right) \mathrm{d} \xi_{4}\right\|_{\mathbb{C}^{4}}^{2} \mathrm{~d} \xi_{1}\right)^{\frac{1}{2}}=0 \\
& \lim _{n \rightarrow \infty}\left(\int\left\|\int f_{n}\left(\xi_{4}\right) \overline{G^{(2)}\left(\xi_{1}, \xi_{4}\right)} U^{(v)}\left(\xi_{4}\right) \mathrm{d} \xi_{4}\right\|_{\mathbb{C}^{4}}^{2} \mathrm{~d} \xi_{1}\right)^{\frac{1}{2}}=0
\end{aligned}
$$

It follows from (4.28), (4.38), (4.44), (4.46) and (4.47) that for every $\epsilon>0$

$$
\limsup _{n \rightarrow \infty}\left\|(H-E-\lambda) \Psi_{n, \epsilon}\right\| \leq 2 \epsilon
$$

This yields

$$
\lim _{\epsilon \rightarrow 0} \limsup _{n \rightarrow \infty}\left\|(H-E-\lambda) \Psi_{n, \epsilon}\right\|=0
$$

In view of (4.49) there exists a subsequence $\left(\Psi_{n_{j}, \epsilon_{j}}\right)_{j \geq 1}$ such that 


$$
\lim _{j \rightarrow \infty}\left\|(H-E-\lambda) \Psi_{n_{j}, \epsilon_{j}}\right\|=0
$$

Furthermore it follows from (4.46) that $w-\lim _{j \rightarrow \infty} \Psi_{n_{j}, \epsilon_{j}}=0$.

The sequence $\left(\Psi_{n_{j}, \epsilon_{j}}\right)_{j \geq 1}$ is a Weyl sequence for $H$ and $E+\lambda$ with $\lambda>0$. In order to show that $E \leq 0$ we adapt the proof given in [5] and [6]. We omit the details.

This concludes the proof of theorem 4.4.

\section{Existence of a Unique Ground State for the Hamiltonian $\boldsymbol{H}$}

Set

$$
\begin{aligned}
& K(F, G)=\sum_{\beta=1}^{2}\left\|F^{(\beta)}(., .)\right\|_{L^{2}}\left\|G^{(\beta)}(., .)\right\|_{L^{2}} \\
& C=2 C_{0} \\
& B=2 m_{n} C_{0}
\end{aligned}
$$

By (4.26) and (5.1) we get for every $\psi \in D(H)$

$$
\left\|H_{I} \psi\right\| \leq K(F, G)\left(C\left\|H_{0} \psi\right\|+B\|\psi\|\right)
$$

In order to prove the existence of a ground state for the Hamiltonian $H$ we shall make the following additional assumptions on the kernels $G^{(\beta)}\left(\xi_{1}, \xi_{4}\right)$, $\beta=1,2$.

From now on $\mathbf{p}_{4} \in \mathbb{R}^{3}$ is the momentum of the neutrino with helicity $-\frac{1}{2}$.

Hypothesis 5.1. There exists a constant $\tilde{K}(G)>0$ such that for $\beta=1,2$ and $\sigma>0$

1) $\int_{\Gamma_{1} \times \mathbb{R}^{3}} \frac{\left|G^{(\beta)}\left(\xi_{1}, \xi_{4}\right)\right|^{2}}{\left|\mathbf{p}_{4}\right|^{2}} \mathrm{~d} \xi_{1} \mathrm{~d} \xi_{4}<\infty$
2) $\left(\int_{\Gamma_{1} \times\left\{\left|\mathbf{p}_{4}\right| \leq \sigma\right\}}\left|G^{(\beta)}\left(\xi_{1}, \xi_{4}\right)\right|^{2} \mathrm{~d} \xi_{1} \mathrm{~d} \xi_{4}\right)^{\frac{1}{2}} \leq \tilde{K}(G) \sigma$

We have

Theorem 5.2. Assume that the kernels $F^{(\beta)}(. .$.$) and G^{(\beta)}(.,),. \beta=1,2$, satisfy Hypothesis 4.1 and Hypothesis 5.1. Then there exists $g_{1} \in\left(0, g_{0}\right]$ such that $H$ has a unique ground state for $g \leq g_{1}$.

In order to prove theorem 5.2 we first prove the existence of a spectral gap for some neutrino infrared cutoff Hamiltonians.

\subsection{The Neutrino Infrared Cutoff Hamiltonians and the Existence of a Spectral Gap}

Proof. Let us first define the neutrino infrared cutoff Hamiltonians.

For that purpose, let $\chi_{0}(.) \in C^{\infty}(\mathbb{R},[0,1])$ with $\chi_{0}=1$ on $(-\infty, 1]$ and $\chi_{0}=0$ on $[2, \infty]$. For $\sigma>0$ and $\mathbf{p}_{4} \in \mathbb{R}^{3}$, we set

$$
\begin{aligned}
& \chi_{\sigma}\left(\mathbf{p}_{4}\right)=\chi_{0}\left(\left|\mathbf{p}_{4}\right| / \sigma\right), \\
& \tilde{\chi}^{\sigma}\left(\mathbf{p}_{4}\right)=1-\chi_{\sigma}\left(\mathbf{p}_{4}\right)
\end{aligned}
$$


The operator $H_{I, \sigma}$ is the interaction given by (4.17) associated with the kernels $F^{(\beta)}\left(\xi_{2}, \xi_{3}\right) \tilde{\chi}^{\sigma}\left(\mathbf{p}_{4}\right) G^{(\beta)}\left(\xi_{1}, \xi_{4}\right)$ instead of $F^{(\beta)}\left(\xi_{2}, \xi_{3}\right) G^{(\beta)}\left(\xi_{1}, \xi_{4}\right)$.

We then set

$$
H_{\sigma}=H_{0}+g H_{I, \sigma}
$$

We now introduce

$$
\begin{aligned}
& \Gamma_{4, \sigma}=\mathbb{R}^{3} \cap\left\{\left|\mathbf{p}_{4}\right|<\sigma\right\}, \quad \Gamma_{4}^{\sigma}=\mathbb{R}^{3} \cap\left\{\left|\mathbf{p}_{4}\right| \geq \sigma\right\} \\
& \mathfrak{F}_{4, \sigma}=\mathfrak{F}_{a}\left(L^{2}\left(\Gamma_{4, \sigma}\right)\right), \quad \mathfrak{F}_{4}^{\sigma}=\mathfrak{F}_{a}\left(L^{2}\left(\Gamma_{4}^{\sigma}\right)\right)
\end{aligned}
$$

$\mathfrak{F}_{4, \sigma} \otimes \mathfrak{F}_{4}^{\sigma}$ is the Fock space for the massless neutrino such that $\mathfrak{F}^{(v)} \simeq \mathfrak{F}_{4, \sigma} \otimes \mathfrak{F}_{4}^{\sigma}$.

We set

$$
\mathfrak{F}^{\sigma}=\mathfrak{F}^{(e)} \otimes \mathfrak{F}^{(p)} \otimes \mathfrak{F}^{(n)} \otimes \mathfrak{F}_{4}^{\sigma} \text { and } \mathfrak{F}_{\sigma}=\mathfrak{F}_{4, \sigma}
$$

We have

$$
\mathfrak{F} \simeq \mathfrak{F}^{\sigma} \otimes \mathfrak{F}_{\sigma}
$$

We further set

$$
H_{0}^{4}=\int\left|\mathbf{p}_{4}\right| b_{+}^{*}\left(\xi_{4}\right) b_{+}\left(\xi_{4}\right) \mathrm{d} \xi_{4}
$$

In the following we identify $H_{0}^{4}$ with its obvious extension to $\mathfrak{F}$.

We let

$$
\begin{aligned}
& H_{0}^{4, \sigma}=\int_{\left|\mathbf{p}_{4}\right| \geq \sigma}\left|\mathbf{p}_{4}\right| b_{+}^{*}\left(\xi_{4}\right) b_{+}\left(\xi_{4}\right) \mathrm{d} \xi_{4}, \\
& H_{0, \sigma}^{4}=\int_{\left|\mathbf{p}_{4}\right|<\sigma}\left|\mathbf{p}_{4}\right| b_{+}^{*}\left(\xi_{4}\right) b_{+}\left(\xi_{4}\right) \mathrm{d} \xi_{4}
\end{aligned}
$$

We identify $H_{0}^{4, \sigma}$ and $H_{0, \sigma}^{4}$ with their obvious extension to $\mathfrak{F}^{\sigma}$ and $\mathfrak{F}_{\sigma}$ respectively.

On $\mathfrak{F}^{\sigma} \otimes \mathfrak{F}_{\sigma}$, we have

$$
H_{0}^{4}=H_{0}^{4, \sigma} \otimes \mathbb{1}_{\sigma}+\mathbb{1}^{\sigma} \otimes H_{0, \sigma}^{4}
$$

where $\mathbb{1}^{\sigma}$ (resp. $\left.\mathbb{1}_{\sigma}\right)$ is the identity operator on $\mathfrak{F}^{\sigma}$ (resp. $\mathfrak{F}_{\sigma}$ ).

Setting

$$
H_{0}^{\sigma}=\left.H_{0}\right|_{\mathfrak{F}^{\sigma}} \text { and } H^{\sigma}=\left.H_{\sigma}\right|_{\mathfrak{F}^{\sigma}}
$$

we then get

$$
\begin{aligned}
& H_{0}^{\sigma}=H_{0, D}^{(e)}+H_{0, D}^{(p)}+H_{0, D}^{(n)}+H_{0}^{4, \sigma} \text { on } \mathfrak{F}^{\sigma} \\
& H^{\sigma}=H_{0}^{\sigma}+g H_{I, \sigma} \text { on } \mathfrak{F}^{\sigma}
\end{aligned}
$$

and

$$
H_{\sigma}=H^{\sigma} \otimes \mathbb{1}_{\sigma}+\mathbb{1}^{\sigma} \otimes H_{0, \sigma}^{4} \quad \text { on } \mathfrak{F}^{\sigma} \otimes \mathfrak{F}_{\sigma}
$$

On the other hand, for $\delta \in \mathbb{R}$ such that $0<\delta<m_{3}$, we define the sequence $\left(\sigma_{n}\right)_{n \geq 0}$ by

$$
\begin{aligned}
& \sigma_{0}=2 m_{e}+1, \\
& \sigma_{1}=m_{e}-\frac{\delta}{2}, \\
& \sigma_{n+1}=\gamma \sigma_{n} \text { for } n \geq 1
\end{aligned}
$$


where

$$
\gamma=1-\frac{\delta}{2 m_{e}-\delta}
$$

For $n \geq 0$, we now introduce the neutrino infrared cutoff Hamiltonians on $\mathfrak{F}^{n}=\mathfrak{F}^{\sigma_{n}}$ by stting

$$
H^{n}=H^{\sigma_{n}}, \quad H_{0}^{n}=H_{0}^{\sigma_{n}}
$$

We set, for $n \geq 0$,

$$
E^{n}=\inf \sigma\left(H^{n}\right)
$$

We introduce the neutrino infrared cutoff Hamiltonians on $\mathfrak{F}$ by setting

$$
H_{n}=H_{\sigma_{n}}, \quad H_{0, n}=H_{0, \sigma_{n}}
$$

We set, for $n \geq 0$,

$$
E_{n}=\inf \sigma\left(H_{n}\right)
$$

Note that

$$
E^{n}=E_{n}
$$

One easily shows that, for $g \leq g_{0}$,

$$
\left|E^{n}\right|=\left|E_{n}\right| \leq \frac{g K(F, G) B}{1-g_{0} K(F, G) C}
$$

See [5] [13] for a proof.

We now let

$$
\tilde{K}(F, G)=2\left(\sum_{\beta=1,2}\left\|F^{(\beta)}(., .)\right\|_{L^{2}\left(\Gamma_{1} \times \Gamma_{2}\right)}\right) \tilde{K}(G)
$$

where $\tilde{K}(G)$ is the constant given in Hypothesis 5.2(2).

We further set,

$$
\begin{aligned}
& \tilde{C}=\frac{C}{1-g_{0} K(F, G) C} \\
& \tilde{B}=\frac{B}{\left(1-g_{0} K(F, G) C\right)^{2}}
\end{aligned}
$$

and

$$
\tilde{D}(F, G)=\max \left\{\frac{4\left(2 m_{3}+1\right) \gamma}{2 m_{3}-\delta}, 2\right\} \tilde{K}(F, G)\left(2 m_{3} \tilde{C}+\tilde{B}\right)
$$

Let $g_{1}^{(\delta)}$ be such that

$$
0<g_{1}^{(\delta)}<\min \left\{1, g_{0}, \frac{\gamma-\gamma^{2}}{3 \tilde{D}(F, G)}\right\}
$$

and let

$$
g_{3}=\frac{1}{2 K(F, G)(2 C+B)}
$$


Setting

$$
\begin{aligned}
& g_{2}^{(\delta)}=\inf \left\{g_{3}, g_{1}^{(\delta)}\right\} \\
& C(F, G)=3 \frac{\tilde{D}(F, G)}{\gamma}
\end{aligned}
$$

and applying the same method as the one used for proving proposition 4.1 in [5] we finally get the existence of a spectral gap for $H^{n}$. We omit the details of the proof.

The proof of the following proposition is achieved.

Proposition 5.3. Suppose that the kernels $F^{(\beta)}(.,),. G^{(\beta)}(.,),. \beta=1,2$, satisfy Hypothesis 4.1 and Hypothesis 5.1(2). Then, for $g \leq g_{2}^{(\delta)}, E^{n}$ is a simple eigenvalue of $H^{n}$ for $n \geq 1$, and $H^{n}$ does not have spectrum in the interval $\left(E^{n}, E^{n}+\left(1-g C(F, G) \sigma_{n}\right)\right)$.

\subsection{Proof of the Existence of a Ground State}

Proof. In order to prove the existence of a ground state for $H$ we adapt the proof of theorem 3.3 in [13]. By Proposition $5.3 H^{n}$ has a unique ground state, denoted by $\phi^{n}$, in $\mathfrak{F}^{n}$ such that

$$
H^{n} \phi^{n}=E^{n} \phi^{n}, \phi^{n} \in \mathcal{D}\left(H^{n}\right),\left\|\phi^{n}\right\|=1, n \geq 1
$$

Therefore $H_{n}$ has a unique normalized ground state in $\mathfrak{F}$, given by $\tilde{\phi}_{n}=\phi^{n} \otimes \Omega_{n}$, where $\Omega_{n}$ is the vacuum state in $\mathfrak{F}_{n}$,

$$
H_{n} \tilde{\phi}_{n}=E^{n} \tilde{\phi}_{n}, \tilde{\phi}_{n} \in \mathcal{D}\left(H_{n}\right),\left\|\tilde{\phi}_{n}\right\|=1, n \geq 1
$$

Let $H_{I, n}$ denote the interaction $H_{I, \sigma_{n}}$. It follows from the pull-through formula that

$$
\begin{aligned}
& \left(H_{0}+g H_{I, n}\right) b_{+}\left(\xi_{4}\right) \tilde{\phi}_{n} \\
& =E_{n} b_{+}\left(\xi_{4}\right) \tilde{\phi}_{n}-\omega\left(\xi_{4}\right) b_{+}\left(\xi_{4}\right) \tilde{\phi}_{n}-\left(g \tilde{V}_{n}^{1}\left(\xi_{4}\right)+g \tilde{V}_{n}^{2}\left(\xi_{4}\right)\right) \tilde{\phi}_{n}
\end{aligned}
$$

where

$$
\begin{aligned}
\tilde{V}_{n}^{(1)}\left(\xi_{4}\right)= & \int \mathrm{d} \xi_{1} \mathrm{~d} \xi_{2} \mathrm{~d} \xi_{3}\left(\int \mathrm{d} x^{2} \mathrm{e}^{-i x^{2} r^{2}}\left(\overline{U^{\left(v_{e}\right)}}\left(\xi_{4}\right) \gamma_{\alpha}\left(1-\gamma_{5}\right) U^{(e)}\left(x^{2}, \xi_{1}\right)\right)\right. \\
& \left.\times\left(\overline{U^{(n e)}}\left(\xi_{3}\right) \gamma^{\alpha}\left(1-g_{A} \gamma_{5}\right) U^{(p)}\left(x^{2}, \xi_{2}\right)\right)\right) \\
& \times \overline{F^{(1)}\left(\xi_{2}, \xi_{3}\right)} \overline{G^{(1)}\left(\xi_{1}, \xi_{4}\right)} \tilde{\chi}^{\sigma_{n}}\left(\mathbf{p}_{4}\right) b_{+}^{*}\left(\xi_{3}\right) b_{+}\left(\xi_{2}\right) b_{+}\left(\xi_{1}\right) \\
\tilde{V}_{n}^{(2)}\left(\xi_{4}\right)= & \int \mathrm{d} \xi_{1} \mathrm{~d} \xi_{2} \mathrm{~d} \xi_{3}\left(\int \mathrm{d} x^{2} \mathrm{e}^{-i x^{2} r^{2}}\left(\overline{U^{\left(v_{e}\right)}}\left(\xi_{4}\right) \gamma_{\alpha}\left(1-\gamma_{5}\right) W^{(e)}\left(x^{2}, \xi_{1}\right)\right)\right. \\
& \times\left(\overline{U^{(n e)}}\left(\xi_{3}\right) \gamma^{\alpha}\left(1-g_{A} \gamma_{5}\right) W^{(p)}\left(x^{2}, \xi_{2}\right)\right) \\
& \times F^{(2)}\left(\xi_{2}, \xi_{3}\right) G^{(2)}\left(\xi_{1}, \xi_{4}\right) \tilde{\chi}^{\sigma_{n}}\left(\mathbf{p}_{4}\right) b_{+}^{*}\left(\xi_{3}\right) b_{-}^{*}\left(\xi_{2}\right) b_{-}^{*}\left(\xi_{1}\right)
\end{aligned}
$$

Hence, by (5.30), (5.31), (5.32) and (5.33), we get

$$
\left(H_{n}-E_{n}+\omega\left(\xi_{4}\right)\right) b_{+}\left(\xi_{4}\right) \tilde{\phi}_{n}=-g\left(\tilde{V}_{n}^{(1)}\left(\xi_{4}\right)+\tilde{V}_{n}^{(2)}\left(\xi_{4}\right)\right) \tilde{\phi}_{n}
$$


We further note that, for $\beta=1,2$,

$$
\left\|\tilde{V}_{n}^{(\beta)}\left(\xi_{4}\right) \tilde{\phi}_{n}\right\| \leq \tilde{C}_{0}\left\|F^{(\beta)}(., .)\right\|_{L^{2}\left(\Gamma_{1} \times \Gamma_{2}\right)}\left\|G^{(\beta)}\left(., \xi_{4}\right)\right\|_{L^{2}\left(\Gamma_{1}\right)} \times\left\|\left(H_{0}+m_{n}\right)^{\frac{1}{2}} \tilde{\phi}_{n}\right\|
$$

where

$$
\tilde{C}_{0}=\left(\frac{1}{m_{n}}\right)^{\frac{1}{2}}\left(\left\|\gamma^{\alpha}\left(1-g_{A} \gamma_{5}\right)\right\|\right)\left(\left\|\gamma_{\alpha}\left(1-\gamma_{5}\right)\right\|\right)
$$

The estimates (5.35) are examples of $N_{\tau}$ estimates (see [20]). The proof is similar to the one of ([21], Proposition 3.7) and details are omitted.

Let us estimate $\left\|H_{0} \tilde{\phi}_{n}\right\|$. By (5.2) we get

$$
g\left\|H_{I, n} \tilde{\phi}_{n}\right\| \leq g K(F, G)\left(C\left\|H_{0} \tilde{\phi}_{n}\right\|+B\right)
$$

and

$$
\left\|H_{0} \tilde{\phi}_{n}\right\| \leq\left|E_{n}\right|+g\left\|H_{I, n} \tilde{\phi}_{n}\right\|
$$

By (5.21), we obtain

$$
\left\|H_{0} \tilde{\phi}_{n}\right\| \leq \frac{g_{0} K(F, G) B}{1-g_{0} K(F, G) C}\left(1+\frac{1}{1-g_{0} K(F, G) C}\right)=M
$$

By (5.38) $\left\|H_{0} \tilde{\phi}_{n}\right\|$ is bounded uniformly with respect to $n$ and $g \leq g_{0}$ and by (5.34), (5.35) and (5.38) we get

$$
\left\|b_{+}\left(\xi_{4}\right) \tilde{\phi}_{n}\right\| \leq \frac{g \tilde{C}_{0}}{\left|\mathbf{p}_{4}\right|}\left(\sum_{\beta=1}^{2}\left\|F^{(\beta)}(., .)\right\|_{L^{2}}\left\|G^{(\beta)}\left(., \xi_{4}\right)\right\|_{L^{2}}\right)\left(M+m_{p}\right)^{\frac{1}{2}}
$$

uniformly with respect to $\mathrm{n}$.

By Hypothesis 5.1(1) and (5.39) there exists a constant $\tilde{C}(F, G)>0$ such that

$$
\int\left\|b_{+}\left(\xi_{4}\right) \tilde{\phi}_{n}\right\|^{2} \mathrm{~d} \xi_{4} \leq \tilde{C}(F, G) g^{2}
$$

Since $\left\|\tilde{\phi}_{n}\right\|=1$, there exists a subsequence $\left(n_{k}\right)_{k \geq 1}$, converging to $\infty$ such that $\left(\tilde{\phi}_{n_{k}}\right)_{k \geq 1}$ converges weakly to a state $\tilde{\phi} \in \mathfrak{F}$. By adapting the proof of theorem 4.1 in [21] it follows from (5.40) that there exists $g_{2}$ such that $0<g_{2} \leq g_{2}^{(\delta)}$ and $\tilde{\phi} \neq 0$ for any $g \leq g_{2}$. Thus $\tilde{\phi}$ is a ground state of $H$.

\subsection{Uniqueness of a Ground State of the Hamiltonian $\boldsymbol{H}$}

Proof. The proof follows by adapting the one given in [6]. See also [25].

In view of theorem $4.3 \mathrm{E}$ is an eigenvalue of $\mathrm{H}$ with a finite multiplicity. Either $E$ is a simple eigenvalue and the theorem is proved or its multiplicity is equal to $p \in \mathbb{N}$ with $p>1$. Let us consider the second case. We wish to show by contradiction that $\mathrm{E}$ is a simple eigenvalue for $\mathrm{g}$ sufficiently small.

Let $\left(\phi_{1}, \phi_{2}\right)$ be two vectors of the eigenspace of E. Each $\phi_{j}$ with $j=1,2$ is a ground state of $H$. $\phi_{1}$ and $\phi_{2}$ can be chosen such that $\left\langle\phi_{1}, \phi_{2}\right\rangle_{\mathfrak{F}}=0$ with $\left\|\phi_{j}\right\|=1, \quad j=1,2$.

By (5.30) let $\phi_{n}$ be a unique normalized ground state of $H_{n}$. 
We have

$$
\begin{aligned}
0 & =\left|\left\langle\phi_{2}, \phi_{1}\right\rangle\right|^{2}=\lim _{n \rightarrow \infty}\left|\left\langle\phi_{2}, \phi_{n}\right\rangle\right|^{2}=\lim _{n \rightarrow \infty}\left\langle E_{\left\{E_{n}\right\}}\left(H_{n}\right) \phi_{2}, \phi_{2}\right\rangle \\
& =1-\lim _{n \rightarrow \infty}\left\langle\left(\mathbb{1}_{\mathfrak{F}}-E_{\left\{E_{n}\right\}}\left(H_{n}\right)\right) \phi_{2}, \phi_{2}\right\rangle
\end{aligned}
$$

where $E_{\{.\}}($.$) is the spectral measure for the associated self-adjoint operator.$

We have

$$
\mathbb{1}_{\mathfrak{F}}-E_{\left\{E_{n}\right\}}\left(H_{n}\right)=\left(\mathbb{1}^{n}-E_{\left\{E_{n}\right\}}\left(H^{n}\right)\right) \otimes P_{\Omega_{n}}+\mathbb{1}^{n} \otimes\left(\mathbb{1}_{n}-P_{\Omega_{n}}\right)
$$

We have to estimate

$$
\left\langle\phi_{2},\left(\mathbb{1}^{n} \otimes\left(\mathbb{1}_{n}-P_{\Omega_{n}}\right)\right) \phi_{2}\right\rangle
$$

and

$$
\left\langle\phi_{2},\left(\left(\mathbb{1}^{n}-E_{\left\{E_{n}\right\}}\left(H^{n}\right)\right) \otimes P_{\Omega_{n}}\right) \phi_{2}\right\rangle
$$

We first estimate (5.43).

By applying the same proof as the one used to get estimates (5.38), (5.39) and (5.40) with $\phi_{2}$ instead of $\tilde{\phi}_{n}$ we easily get

$$
\int\left\|b_{+}\left(\xi_{4}\right) \phi_{2}\right\|^{2} \mathrm{~d} \xi_{4} \leq \tilde{C}(F, G) g^{2}
$$

This yields

$$
\left\langle\phi_{2},\left(\mathbb{1}^{n} \otimes\left(\mathbb{1}_{n}-P_{\Omega_{n}}\right)\right) \phi_{2}\right\rangle \leq \tilde{C}(F, G) g^{2}
$$

We now estimate (5.44)

Set

$$
\mathbb{1}^{n}-E_{\left\{E_{n}\right\}}\left(H^{n}\right)=E_{\left\{E_{n}\right\}}\left(H^{n}\right)^{\perp}
$$

By proposition 5.3 we get

$$
\left(H^{n}-E_{n}\right) E_{\left\{E_{n}\right\}}\left(H^{n}\right)^{\perp} \geq(1-g C(F, G)) \sigma_{n} E_{\left\{E_{n}\right\}}\left(H^{n}\right)^{\perp}
$$

and

$$
\begin{aligned}
& \left\langle\phi_{2}, E_{\left\{E_{n}\right\}}\left(H^{n}\right)^{\perp} \otimes P_{\Omega_{n}} \phi_{2}\right\rangle \\
& \leq \frac{1}{(1-g C(F, G)) \sigma_{n}}\left\langle\phi_{2},\left(H^{n}-E_{n}\right) \otimes P_{\Omega_{n}} \phi_{2}\right\rangle \\
& =\frac{1}{(1-g C(F, G)) \sigma_{n}}\left\langle\phi_{2},\left(H_{n}-E_{n}\right)\left(\mathbb{1}^{n} \otimes P_{\Omega_{n}}\right) \phi_{2}\right\rangle \\
& \leq \frac{1}{(1-g C(F, G)) \sigma_{n}}\left\langle\phi_{2},\left(H_{n}-E_{n}\right) \phi_{2}\right\rangle
\end{aligned}
$$

Note that

$$
E \leq\left\langle\tilde{\phi}_{n}, H \tilde{\phi}_{n}\right\rangle=\left\langle\phi_{n}, H^{n} \phi_{n}\right\rangle=E^{n}=E_{n}
$$

In view of (5.49) and of (5.50) we get 


$$
\begin{aligned}
& \frac{\left\langle\phi_{2},\left(H_{n}-E_{n}\right) \phi_{2}\right\rangle}{(1-g C(F, G)) \sigma_{n}} \\
& =\frac{\left\langle\phi_{2},\left(E-E_{n}\right) \phi_{2}\right\rangle}{(1-g C(F, G)) \sigma_{n}}+\frac{\left\langle\phi_{2},\left(H_{n}-H\right) \phi_{2}\right\rangle}{(1-g C(F, G)) \sigma_{n}} \\
& \leq \frac{\left\langle\phi_{2},\left(H_{n}-H\right) \phi_{2}\right\rangle}{(1-g C(F, G)) \sigma_{n}}
\end{aligned}
$$

Hence

$$
\left\langle\phi_{2}, E_{\left\{E_{n}\right\}}\left(H^{n}\right)^{\perp} \otimes P_{\Omega_{n}} \phi_{2}\right\rangle \leq \frac{\left\langle\phi_{2},\left(H_{n}-H\right) \phi_{2}\right\rangle}{\left(1-g_{2}^{(\delta)} C(F, G)\right) \sigma_{n}}
$$

Here $g_{2}^{(\delta)}$ has been introduced in proposition 5.3

Estimate of $\left\|\left(H_{n}-H\right) \tilde{\phi}\right\|$. We have

$$
H-H_{n}=g\left(H_{I}-H_{I, n}\right)
$$

$H-H_{n}$ is associated with the kernels $F^{(\beta)}\left(\xi_{2}, \xi_{3}\right) \chi_{\sigma_{n}}\left(\mathbf{p}_{4}\right) G^{(\beta)}\left(\xi_{1}, \xi_{4}\right)$.

By adapting the proof of (5.2) to the estimate of $\left(H-H_{n}\right)$ we finally get

$$
\left\|\left(H-H_{n}\right) \phi_{2}\right\|=g\left\|\left(H_{I}-H_{I, n}\right) \phi_{2}\right\| \leq g K_{n}(F, G)\left(C\left\|H_{0} \phi_{2}\right\|+B\right)
$$

where

$$
K_{n}(F, G)=\sum_{\beta=1}^{2}\left\|F^{(\beta)}(., .)\right\|_{L^{2}}\left\|\chi_{\sigma_{n}}\left(\mathbf{p}_{4}\right) G^{(\beta)}(., .)\right\|_{L^{2}}
$$

Under Hypothesis 5.2(2) we get

$$
K_{n}(F, G) \leq 2\left(\sum_{\beta=1}^{2}\left\|F^{(\beta)}(., .)\right\|_{L^{2}}\right) \tilde{K}(G) \sigma_{n}
$$

This, together with (5.55), yields

$$
\left|\left\langle\phi_{2},\left(H-H_{n}\right) \phi_{2}\right\rangle\right| \leq g K \sigma_{n}
$$

where $K=2\left(\sum_{\beta=1}^{2}\left\|F^{(\beta)}(., .)\right\|_{L^{2}}\right) \tilde{K}(G)\left(C\left\|H_{0} \phi_{2}\right\|+B\right)$.

Combing (5.41), (5.42), (5.46), (5.52) and (5.57) we finally get

$$
\left\langle\phi_{2},\left(\mathbb{1}_{\mathfrak{F}}-E_{\left\{E_{n}\right\}}\left(H_{n}\right)\right) \phi_{2}\right\rangle \leq g K^{\prime}
$$

Here $K^{\prime}=\frac{K}{1-g_{2}^{(\delta)} C(F, G)}$.

$K^{\prime}$ is a positive constant independent of $g$ and it follows from (5.41) that, for $g$ sufficiently small, $\left\langle\phi_{1}, \phi_{2}\right\rangle \neq 0$. This is a contradiction and $p=1$. This concludes the proof of theorem 5.2.

\section{Acknowledgements}

J.-C. G. acknowledges J.-M Barbaroux, J. Faupin and G. Hachem for helpful discussions. 


\section{References}

[1] Bhattacharya, K. and Pal, P.B. (2004) Inverse Beta Decay of Arbitrarily Polarized Neutrons in a Magnetic Field. Pramana, 62, 1041. https://doi.org/10.1007/BF02705251

[2] Bhattacharya, K. and Pal, P.B. (2004) Neutrinos and Magnetic Fields: A Short Review. Proceedings of the National Academy of Sciences, 70, 145.

[3] Duan, H. and Qian, Y.Z. (2005) Rates of Neutrino Absorption on Neutrons and the Reverse Processes in Strong Magnetic Fields. Physical Review D, 72, Article ID: 023005. https://doi.org/10.1103/PhysRevD.72.023005

[4] Guinti, C. and Studenikin, A. (2015) Neutrino Electromagnetic Interactions: A Window to New Physics. Reviews of Modern Physics, 87, 531. https://doi.org/10.1103/RevModPhys.87.531

[5] Aschbacher, W.H., Barbaroux, J.-M., Faupin, J. and Guillot, J.-C. (2011) Spectral Theory for a Mathematical Model of the Weak Interaction: The Decay of the Intermediate Vector Bosons $W^{ \pm}$. II. Annales Henri Poincaré, 12, 1539-1570. https://doi.org/10.1007/s00023-011-0114-3

[6] Barbaroux, J.-M., Faupin, J. and Guillot, J.-C. Local Decay for Weak Interactions with Massless Particles. ArXiv 1611.0 7814. To Be Published in Journal of Spectral Theory.

[7] Thaller, B. (1992) The Dirac Equation. Texts and Monographs in Physics, Springer Verlag, Berlin.

[8] Hachem, G. (1993) Effect Zeeman pour un électron de Dirac. Annales de l'Institut Henri Poincaré, 58, 105-123.

[9] Johnson, M.H. and Lippmann, B.A. (1950) Relativistic Motion in a Magnetic Field. Physical Review, 77, 702-705. https://doi.org/10.1103/PhysRev.77.702

[10] Itzykson, C. and Zuber, J.-B. (1980) Quantum Field Theory. McGraw-Hill Book Company.

[11] Bhattacharya, K. (2007) Solution of the Dirac Equation in Presence of an Uniform Magnetic Field. ArXiv 0705.4275

[12] Weinberg, S. (2005) The Quantum Theory of Fields. Vol. I. Cambridge University Press, Cambridge.

[13] Barbaroux, J.-M. and Guillot, J.-C. (2009) Spectral Theory for a Mathematical Model of the Weak Interaction: The Decay of the Intermediate Vector Bosons $W^{ \pm}$. I. Advances in Mathematical Physics, Article ID: 978903.

[14] Dereziński, J. and Gérard, C. (1999) Asymptotic Completeness in Quantum Field Theory. Massive Pauli-Fierz Hamiltonians. Reviews in Mathematical Physics, 11, 383-450. https://doi.org/10.1142/S0129055X99000155

[15] Reuse, F.A. (2007) Electrodynamique et Optiques Quantiques. Presses Polytechniques et Universitaires Romanes, Lausanne.

[16] Guillot, J.C. (2015) Spectral Theory of a Mathematical Model in Quantum Field Theory for Any Spin. Contemporary Mathematics, 640, 13-37. https://doi.org/10.1090/conm/640/12842

[17] Greiner, W. and Müller, B. (1989) Gauge Theory of Weak Interactions. Springer, Berlin.

[18] Weinberg, S. (2005) The Quantum Theory of Fields. Vol. II. Cambridge University Press, Cambridge.

[19] Beranger, J., et al. (Particle Data Group) (2012) Physical Review D, 86, O10001. 
[20] Glimm, J. and Jaffe, A. (1985) Quantum Field Theory and Statistical Mechanics. Birkhäuser, Boston Inc., Boston.

[21] Barbaroux, J.-M., Dimassi, M. and Guillot, J.-C. (2004) Quantum Electrodynamics of Relativistic Bound States with Cutoffs. Journal of Hyperbolic Differential Equations, 1, 271-314. https://doi.org/10.1142/S021989160400010X

[22] Dereziński, J. and Gérard, C. (2013) Mathematics of Quantization and Quantum Fields. Cambridge University Press. https://doi.org/10.1017/CBO9780511894541

[23] Arai, A. (2000) Essential Spectrum of a Self-Adjoint Opeator on a Abstract Hilbert of Fock Type and Applications to Quantum Field Halmitonians. Journal of Mathematical Analysis and Applications, 246, 189-216.

https://doi.org/10.1006/jmaa.2000.6782

[24] Takaesu, T. (2014) Essential Spectrum of a Fermionic Quantum Field Model. Infinite Dimensional Analysis, Quantym Probability and Related Topics, 17, 1450024. https://doi.org/10.1142/S0219025714500246

[25] Bach, V., Fröhlich, J. and Sigal, I. (1998) Quantum Electrodynamics of Confined Relativistic Particles. Advances in Mathematics, 137, 299-395.

https://doi.org/10.1006/aima.1998.1734 